\title{
Im Reich des Augeias: Elis und Olympia zwischen 1200 und 700 v. Chr. ${ }^{1}$
}

Augeias kennt man vor allem wegen seiner sprichwörtlich gewordenen Ställe, die auszumisten eine der zwölf Taten des Herakles war. Im Mittelpunkt der folgenden Überlegungen steht allerdings die Landschaft Elis in der nordwestlichen Peloponnes, als deren Herrscher in der Ilias Homers $(11,702)$ Augeias genannt wird. Der vorliegende Beitrag behandelt in erster Linie archäologische Zeugnisse aus der antiken Stadt Elis und aus dem Heiligtum von Olympia, welche den früheisenzeitlichen Perioden Griechenlands (11.-8. Jh. v. Chr.) angehören. Meiner Auffassung nach bietet die frühe Eisenzeit in vielem den historischen Hintergrund für die Epen Homers, und insofern findet der mythische Bezug im Titel meines Beitrags auf Augeias seine Berechtigung. Im folgenden möchte ich nicht nur eine Zusammenfassung der Ergebnisse meiner langjährigen Beschäftigung mit Funden aus Elis und Olympia bieten, sondern auch einen Überblick über die Entwicklungen in der Periode zwischen dem Ende der Spätbronzezeit und dem Beginn der Eisenzeit im Westen des griechischen Festlands vermitteln. Es war erst die Auseinandersetzung mit vielen Detailproblemen, die das Verständnis für die größeren $\mathrm{Zu}$ sammenhänge innerhalb einer bislang unzureichend erforschten Landschaft und ihre Einbindung in den Kosmos der westgriechischen Welt in einer historischen Umbruchsphase entstehen ließ.

Während der Spätbronzezeit war die Landschaft Elis relativ dicht besiedelt, aber ein mykenischer Palast, der dieses Gebiet beherrschte, wurde bisher nicht gefunden, und es existieren auch keine Hinweise darauf, daß ein solcher tatsächlich einmal existierte. Anders als in anderen Regionen des myke-

${ }^{1}$ Der vorliegende Text stellt die überarbeitete Fassung eines Vortrags dar, welcher am 13. 3. 2002 an der Universität Wien und am 8. 5. 2002 am Deutschen Archäologischen Institut in Berlin gehalten wurde. An dieser Stelle bietet sich mir die Gelegenheit, meinen Dank an all jene zu formulieren, die meine Beschäftigung mit der Frühzeit der westlichen Peloponnes und insbesondere der Landschaft Elis, ermöglicht und gefördert haben. Durch Veronika Mitsopoulos-Leon und Nikolas Yalouris, die mir die Publikation der früheisenzeitlichen Gräber aus den griechisch-österreichischen Grabungen in der antiken Stadt Elis übertrugen, eröffnete sich mir die Beschäftigung mit der Frühzeit dieses doch recht unbekannten Landstrichs. Die Hilfsbereitschaft und freundschaftliche Unterstützung durch Kollegen des griechischen Antikendienstes, namentlich Georgia Chatzi-Spiliopoulou, Theodora Karagiorga, Georgios Papathanassopoulos und Konstantinos Zachos ermöglichten mir, einige andere, ebenfalls unpublizierte Fundgruppen in meine Studien einzubeziehen. Danken möchte ich in diesem Zusammenhang auch der Ephorin von Olympia, Xeni Arapogianni, und Olympia Vikatou für ihre dauerhafte Unterstützung meiner Arbeiten in Elis und Olympia. Das Angebot von Helmut Kyrieleis, die früheisenzeitliche Keramik aus seinen Grabungen am Pelopion in Olympia zu bearbeiten und zu veröffentlichen, verhalf mir dann zu olympischen Ehren. In diesem Zusammenhang möchte ich dankbar die Unterstützung durch ein APART-Stipendium der Österreichischen Akademie der Wissenschaften hervorheben, in dessen Verlauf wichtige Abschnitte an den genannten Arbeiten entstanden sind. Sigrid Deger-Jalkotzy danke ich für die Anregung zu dieser Publikation und für die Vorlage des Manuskripts in der Sitzung der phil.-hist. Klasse der Akademie. 
nischen Griechenlands fehlen reich ausgestattete Gräber oder die architektonisch auffälligen Kuppelgräber, welche die Konzentration von Macht während der frühmykenischen Zeit oder während der mykenischen Palastzeit des 14. und 13. Jhs. nahelegen².

Es ist anzunehmen, daß die Region während der mykenischen Palastzeit in einem gewissen Spannungsverhältnis zum weiter südlich gelegenen Reich von Pylos stand. Die Nordgrenze des mykenischen Messenien wird seit J. Chadwick traditionell entlang des Flußlaufs der Neda gezogen, hauptsächlich aufgrund rein geographischer Gesichtspunkte. Angesichts der Erwähnung von Ethnika bzw. Herkunftsbezeichnungen und Ortsnamen in den Linear BTexten von Pylos, die mögliche Bezüge zur Insel Zakynthos, zu Pisa, Olympia, zum Fluß Erymanthos und dem elischen(?) Ethnikon der Metapier herstellen, bleibt zu fragen, ob das Einflußgebiet des Reiches von Pylos nicht einst gröBer war als bislang vermutet und bis zum Alpheiostal reichte ${ }^{3}$. Nimmt man an, daß Triphylien und das Alpheiostal tatsächlich einmal Teil des mykenischen Reichs von Pylos waren, wird verständlich, warum dieser Landstrich in der Ilias Homers $(2,591$ ff.) noch stellvertretend für den Rest Messeniens als Herrschaftsgebiet des Nestor angesprochen werden kann. Die zentralen Gebiete des messenischen Palastsstaates dies- und jenseits des Aigaleonberges weisen tasächlich Anzeichen eines Siedlungsbruches nach dem Ende des Palastes von Pylos um 1200 v. Chr. auf, der sich u. a. im Fehlen einer Ortsnamenkontinuität ausdrückt ${ }^{4}$. Archäologische Daten und das angesprochene Namensmaterial legen hingegen eine stärkere Siedlungskontinuität im Alpheiostal nahe.

Die mykenische Besiedlung der Landschaft Elis konzentrierte sich entlang der beiden großen Flußtäler der Region, im Bereich des Peneios im Norden und vor allem im Bereich des Alpheios im Süden. Die Siedlungsdichte läßt sich hauptsächlich anhand von mykenischen Friedhöfen erschließen, welche die Mehrzahl aller mykenischen Fundplätze innerhalb der Landschaft bil-

2 Anders stellt sich die Situation im benachbarten Triphylien oder Messenien dar, wo die Tholosgräber von Kakovatos und Peristeria mit ihrer Ausstattung auf die Existenz frühmykenischer Eliten innerhalb eines geographisch begrenzten Raumes schließen lassen. Dickinson 1977, 92 f. Vgl. CavanaGH - MeE 1998, 56; zur entsprechenden Entwicklung in Messenien vgl. zuletzt BENNET 1999.

3 Diese Gedanken sind nicht völlig neu, sondern sind immer wieder in der einschlägigen Literatur aufgetaucht, haben bislang allerdings keine Mehrheit gefunden. J. Chadwicks große wissenschaftliche Autorität hat hier sicherlich prägend gewirkt, auch wenn sich seine Argumente im Fall der Nordgrenze hauptsächlich auf die große geographische Distanz des Alpheiostals von Pylos berufen. Die Identifizierung der einzelnen Linear B-Namen mit bekannten Toponymen und Ethnika der klassischen Zeit ist in der Forschung umstritten. Immerhin wird es in der Forschung für möglich gehalten, daß im Text PY An 1, der die Entsendung von Ruderern nach Pleuron anspricht, der entsprechende Ort in Aitolien gemeint ist. Zakynthos und Krokyleia gehören im Schiffskatalog der Ilias (2, 631 ff.) zum Reich des Odysseus. Ausgewählte Literatur zum umfangreichen Thema der mykenischen Geographie von Pylos: VentRIs - CHadwick 1973, 139-145. 415-417; CHADWICK 1972; HILler 1972, bes. 50 f.; PARKER 1993; siehe außerdem die bibliographischen Angaben zu den verschiedenen Interpretationen bei AURA-Jorro - Adrados 1999, s. v. ko-ro-ku-ra-i-jo, me-ta-pa; AURA-Jorro - AdRAdos 1993, s. v. o-ru-ma-to, pi-*82, pe-re-u-ro-na-de, u-ru-pi-ja-jo, za-ku-si-ja, za-ku-si-jo. Neuere Arbeiten zur pylischen Geographie bei Bennet 1998; Bennet 1998-99; Bennet 1999.

4 Siehe EDER 1998, 144f., 184-186. Der homerische Schiffskatalog spiegelt die politische Geographie nachmykenischer Zeit, vgl. EDER 2003. 
$\operatorname{den}^{5}$. Der Zusammenbruch der mykenischen Paläste in anderen Landschaften der Peloponnes um 1200 v. Chr. stellte in Elis kein so einschneidendes Ereignis dar wie etwa in der südlich angrenzenden Landschaft Messenien, wo nicht nur der Palast von Pylos und sein bürokratisches System unwiederbringlich ihr Ende fanden, sondern auch eine große Zahl von Orten anscheinend verlassen wurde ${ }^{6}$.

Die Landschaft Elis in der Postpalatialen Zeit des 12. und FrÜHen 11. Jhs. v. Chr. (Авв. 1)

Während der spätmykenischen Periode SH III C (ca. 1200-1070/50 v. Chr.), setzte sich die Besiedlung in Elis kontinuierlich fort, und diese nachpalatiale Periode SH III C war hier - allem Anschein nach - eine Zeit der Blüte und des Wohlstands. Entsprechende Funde kommen etwa aus mykenischen Kammergräbern im Peneiostal. So zeigen Keramikfunde aus einem Kammergrab bei

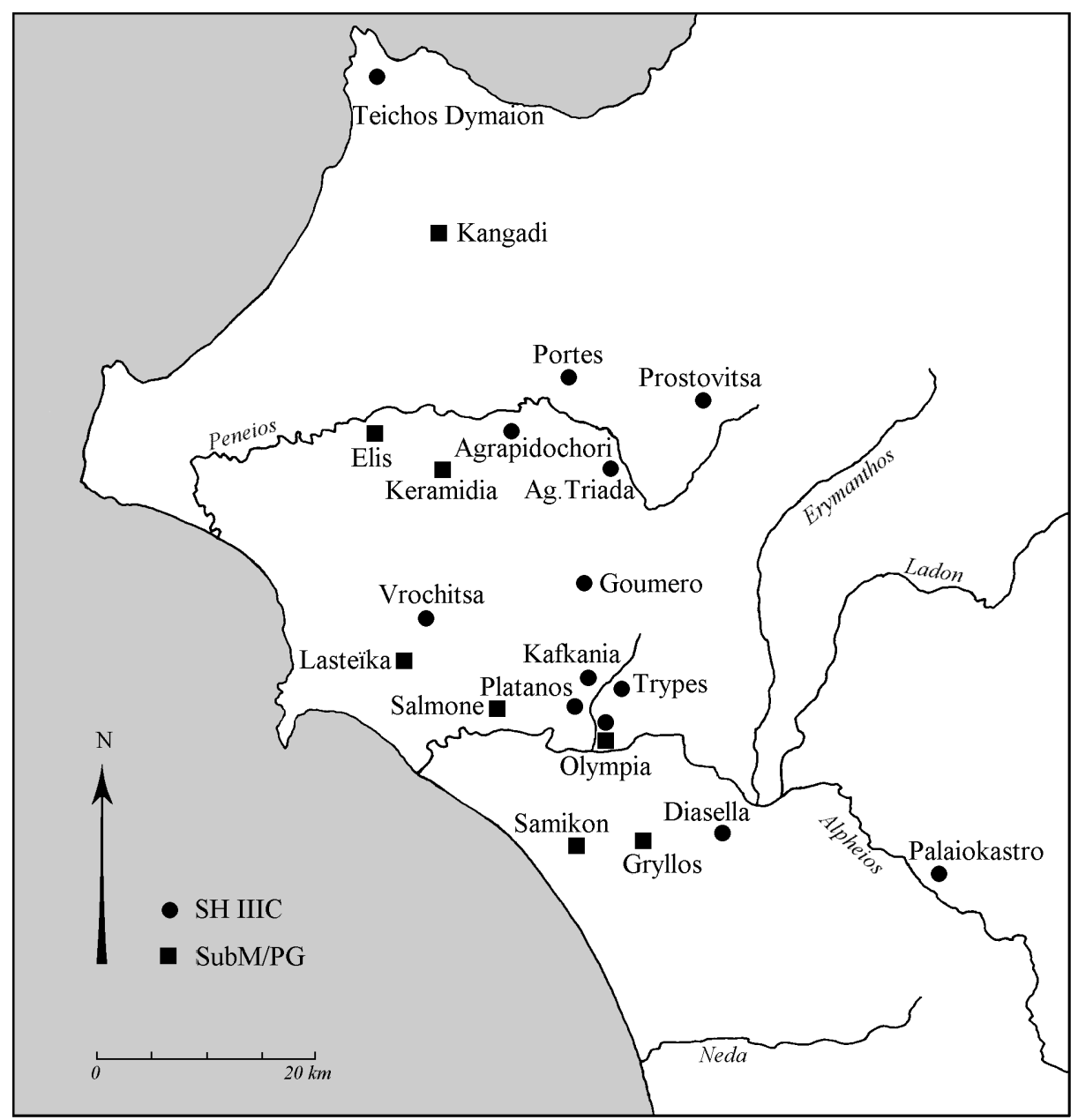

Abb. 1: Karte der Landschaft Elis mit Fundorten des 12. bis 10. Jhs. v. Chr.

5 Mykenische Besiedlung von Elis: Hope Simpson 1981, 92-96 D 48-70, 153-155 G 1-6; Parlama 1974; ferner Mountjoy 1999, 365-398. Zu Neufunden siehe Vikatou 1999a; Vikatou 1996-97.

${ }^{6}$ Messenien und das Ende des Palastes von Pylos: Shelmerdine 2001, 372-376; Eder 1998, 141-162. 
Agrapidochori an der Stelle, wo der Fluß Ladon in den Peneios mündet, daß auch hier die Besiedlung der Region bis zum Ende der Periode SH IIIC Spät fortdauerte7. Vor allem anhand der Keramik lassen sich Kontakte zwischen Elis und der nördlichen Nachbarlandschaft Achaia veranschaulichen, denn die besten Parallelen für die Keramik aus Agrapidochori lassen sich eben in Achaia finden. Tatsächlich sind Grenzen zwischen dem westlichen Achaia und dem nördlichen Elis anhand der bronzezeitlichen Sachkultur nicht faßbar. Ähnliches gilt für Funde der Periode SH IIIC, die in den letzten Jahren in Agia Triada ausgegraben wurden ${ }^{8}$. Sie stammen aus einer reich ausgestatteten mykenische Nekropole, die am Oberlauf des Peneios an einer verkehrstechnisch interessanten Stelle liegt, wo sich die Ost-West-Verbindungen entlang des Flußlaufes mit dem Landweg aus dem Norden der Peloponnes kreuzen. Die Kammergräber dieses Friedhofs wurden nach Ausweis der Funde anscheinend besonders intensiv während SH IIIC, jedenfalls aber bis zum Ende dieser letzten mykenischen Epoche, d. h. bis in das erste Drittel des 11. Jhs. v. Chr. als Grabstätten benutzt.

Aus dem Dromos eines dieser Gräber stammt ein Krater mit der Darstellung einer Prothesis-Szene aus SH IIIC. Dieses Gefäß, das zusammen mit Scherben von Kylikes und Schalen gefunden wurde, weist auf Trinkrituale hin, welche im Zusammenhang mit den Begräbnisfeierlichkeiten vor der eigentlichen Grabkammer im Dromos stattfanden. Ähnlich wie bei Bildern, die von attischen Vasen aus der zweiten Hälfte des 8 . Jhs. bekannt sind, liegt hier im Zentrum der Darstellung der Verstorbene auf seiner Kline, darunter ein Hund. Links und rechts der Bahre bewegen sich Figuren, die im Trauergestus mit ihren Händen den Kopf berühren ${ }^{9}$. Vergleichbare Szenen erscheinen auch auf den mykenischen Tonsärgen aus der Nekropole von Tanagra, die wahrscheinlich der Zeitspanne des 14.-12. Jhs. v. Chr. angehören ${ }^{10}$. Die Ähnlichkeiten zwischen mykenischen und geometrischen Darstellungen liegt anscheinend darin begründet, daß der Grabritus über die Jahrhunderte ganz ähnlich und gleichbleibend verlaufen ist. Dies ist ein bemerkenswerter Zug an Kontinuität von sozialen Verhaltensmustern in einer Zeit, deren Charakter als Periode radikaler Veränderungen in der Forschung immer noch stark im Vordergrund steht. Tatsächlich ist aber damit zu rechnen, daß in der Zeit nach dem Fall der mykenischen Paläste regionale Eliten bis zu einem gewissen Grad die Kontinuität sozialer Strukturen und Verhaltensweisen aus der Zeit der mykenischen Paläste gewährleisten konnten. Dazu gehörte auch der Aufwand, den ein standesgemäßes Begräbnis mit Salbung des Leichnams, Aufbahrung, Totenklage, Bestattung, möglicherweise auch Wettkämpfen, erforderte. Diese Begräbnisriten waren, wenn man die Ähnlichkeiten der bildlichen Darstellungen des 13. und 12. Jhs. v. Chr. einerseits und jene des ausgehenden 8. Jhs. sowie die poetische Beschreibung homerischer Begräbnisse andererseits als entsprechende Hinweise wertet, über die Jahrhunderte von 1300-700 v. Chr. kontinuierliche Sitte geblieben ${ }^{11}$. Solche Bilder von Begräbnisszenen sind zwar aus der Zeit zwischen dem 11. und 10. Jh. v. Chr. nicht be-

7 Agrapidochori: Parlama 1971; MountJoy 1999, 379-397 Elis Nr. 32, 42, 44, 48, 52, 55, $61,65,70,77,79,80,82,84,87,90,95,99,100$.

8 Agia Triada: VIKatou 1999b; bibliographische Verweise auf die Vorberichte bei EDER 2001a, 235 Anm. 5.

9 ViKatou 2001.

10 Für Referenzen siehe Cavanagh - Mee 1995; Immerwahr 1995.

11 Cavanagh - Mee 1995, 58f.; Cavanagh 1998, 105-113; Rysted 1999. Zu mykenischen Totenagonen: DECKER 1987. 
kannt, allerdings zeichnet sich diese Periode auf dem griechischen Festland in großem Maße durch das Fehlen von bildlichen Darstellungen aus. Der Krater aus Agia Triada bildet insofern eine wichtiges Beispiel in der Dokumentation der Kontinuität sozialer Verhaltensformen von der mykenischen Zeit bis in die historische Epoche Griechenlands.

Im Süden der Landschaft, im Alpheiostal, bezeugen ebenfalls Funde Kontinuität über das Ende der mykenischen Palastzeit hinweg. Beispiele stammen etwa aus den Gräbern von Kafkania ${ }^{12}$ und der Nekropole von Trypes im oberen Kladeostal. SH IIIC Gefäße wie ein Alabastron oder eine Amphora mit zwei Horizontal- und zwei kleineren Vertikalhenkeln aus dieser Nekropole bei Trypes sind charakteristisch für die Keramik der Region und weisen deutliche Parallelen zu zeitgleichen Funden aus dem großen mykenischen Kammergrabfriedhof von Palaiokastro auf, der am Oberlauf des Alpheios im heutigen Verwaltungsbezirk Arkadien liegt ${ }^{13}$. Das Tal des Alpheios bildete hier offensichtlich eine Kontaktzone und ermöglichte Verbindungen vom Westen des Landes nach Osten ins Landesinnere. Ähnlichkeiten zwischen den Funden aus der spätmykenischen Nekropole von Palaiokastro in Arkadien und jener von Pellana in Lakonien lassen darüber hinaus vermuten, daß in der Spätbronzezeit zwischen Elis und Lakonien eine Landroute durch die Berge der Westpeloponnes über das Becken von Megalopolis entlang der Flußtäler des Alpheios und des Eurotas verlief.

In Olympia bezeugen mykenische Kammergräber, die hinter dem neuen Museum ausgegraben wurden, daß hier eine kleine mykenische Siedlung in der unmittelbaren Nachbarschaft existierte. Die Keramik aus diesen Gräbern legt nahe, daß die mykenische Besiedlung von Olympia bis in die Periode $\mathrm{SH}$ IIIC Früh reichte, d. h. bis in das frühe 12. Jh. v. Chr., danach endet die Nutzung dieser Gräber ${ }^{14}$. Es gibt keine Hinweise auf kontinuierliche Besiedlung bis in die frühe Eisenzeit. Es fehlen alle klaren Hinweise auf Kultaktivitäten, auch wenn eine Kultstätte für das mykenische Dorf, analog zum gängigen Befund mykenischer Siedlungen, grundsätzlich zu postulieren ist.

Im Zusammenhang mit dieser Siedlung müssen auch einige Scherben verstanden werden, welche im heiligen Bezirk von Olympia gefunden wurden ${ }^{15}$. Mykenische Scherben in sehr geringem Ausmaß stammen aus sekundären Ablagerungen im Stadion-Nordwall, wo dieser in den Südostabhang des Kronoshügels übergeht. Zu den Funden gehören auch die Köpfchen zweier

12 Kafkania: Parlama 1974, 33-35, 42-45; MountJoy 1999, 395-397 Nr. 85. 89. 92.93. 96.

13 Mykenische Nekropole von Kladeos-Trypes/Stravokephalo im Kladeostal: siehe bibliographische Verweise bei EdER 2001a, 234f. Anm. 2; MounTJoy 1999, 391-397 Nr. 73-76, 78, 81, 83, 88, 97 (SH IIIC). Zu Palaiokastro im oberen Alpheiostal zuletzt DEMAKOPOULOU - CROUWEL 1998.

14 Mykenisches Olympia: Siehe Bibliographie bei Eder 2001a, 235 Anm. 3; Eder 2001b, Anm. 15.

$15 \mathrm{Zu}$ den mykenischen Funden aus der Altis und dem Stadion-Nordwall vgl. HeRRMANN 1962, 24, Beilage 4.1-2; HeRrmann 1987. Er vertritt allerdings in der Tradition von W. Dörpfeld die Auffassung von einem prähistorischen Ursprung des späteren Zeusheiligtums und -kults und interpretiert die verstreuten mykenischen Keramikfunde in diesem Sinne. Vgl. hingegen Eder 2001b, 202f. und EdER, Pelopion. Die bemalte und unbemalte mykenische Keramik aus diesem Gebiet, von der nur wenige Stücke veröffentlicht sind, gehört den Perioden SH IIA-IIIB an. Sie umfaßt vor allem offene Gefäßtypen, die durch Stiele und Füße mykenischer Kylikes repräsentiert sind. Eine eingehenden Studie der mykenischen Keramik aus Olympia ist von der Verfasserin für die Zukunft vorgesehen. 
mykenischer Figurinen ${ }^{16}$. Die mykenischen Funde erstrecken sich über das Gebiet vom Osten des Stadion-Nordwalls über die Schatzhausterrasse bis weiter westlich in das Gebiet des Prytaneions ${ }^{17}$. Die Fundorte dieser verstreuten, einzelnen Keramikfragmente in sekundären Ablagerungen legen eine Nutzung des Geländes auf oder an den Hängen des Kronoshügels in mykenischer Zeit nahe, über deren Charakter anhand der Funde keine Aussage möglich ist. Angesichts der verfügbaren Evidenz kann die ursprüngliche Existenz einer mykenischen Kultstätte auf dem Kronoshügel nicht völlig ausgeschlossen werden. Natürlich gibt es aber auch keine tragfähige Grundlage für eine derartige Annahme, denn charakteristische Funde eines mykenischen Heiligtums wie etwa scheibengedrehte Stier- und Menschenfiguren fehlen ${ }^{18}$. Die bescheidenen Befunde sind für weitergehende Interpretationen nicht aussagekräftig genug. Die Zeugnisse mykenischer Zeit in Olympia stehen sowohl chronologisch als auch topographisch in keinem unmittelbaren Zusammenhang mit jenen, die aus jüngeren Epochen im heiligen Bezirk zutage gekommen sind. Insofern fehlt jeder Hinweis, um den Anspruch auf Kultkontinuität des späteren Heiligtums aus der Bronzezeit zu begründen.

Soweit sich die gesamte Situation innerhalb der Region vorläufig beurteilen läßt, fanden während der Periode SH IIIC Spät die letzten Beisetzungen in den mykenischen Kammergrabnekropolen der Landschaft Elis statt. Nach Ausweis der Funde wurden diese Begräbnisstätten nach dem Ende der Periode SH IIIC nicht mehr weiterbelegt, sondern aufgegeben. In einer absoluten Chronologie läßt sich das Ende der Periode SH IIIC mit aller Vorsicht zwischen 1070-1050 v. Chr. festlegen. Diese Zäsur muß mit einer Veränderung des Siedlungsmusters innerhalb der ganzen Region Hand in Hand gegangen sein, denn die Aufgabe der jahrhundertelang genutzten Begräbnisstätten der mykenischen Zeit am Ende der Periode SH IIIC betrifft alle bislang bekannten Fundplätze in der Landschaft. Dieses Phänomen ist auch in anderen Landschaften der Peloponnes beobachtet worden. Sowohl in der Argolis, in Lakonien und in Messenien, aber auch in Achaia und in der Korinthia sind auffällige Veränderungen in der Wahl von Siedlungs- und Begräbnisplätzen zwischen SH IIIC und der protogeometrischen Zeit (etwa in der Mitte des 11. Jhs. v. Chr.) festgestellt worden ${ }^{19}$. Dieser archäologisch faßbare Bruch am Ende der Periode SH IIIC scheint aber auf das südgriechische Festland beschränkt zu sein, denn er ist weder für den nord- und mittelgriechischen Raum noch für Kreta und Zypern in vergleichbarer Weise zu beobachten. Es ist damit zu rechnen, daß in dieser Periode in den ehemaligen Kernlandschaf-

16 HeilmeYer 1979, 9 Taf. 2 Nr. 1-2.

17 Siehe auch oben Anm. 15. Westlich des Stadions wurde 1977 am Fuß des Kronoshügels ein Schnitt hinter der Terrassenmauer der Schatzhausterrasse angelegt. Aus den Aufschüttungen hinter der Mauer stammen der Fuß und ein Wandfragment eines mykenischen Goblets, welcher den Periode SH III A-B zuzuweisen sein dürfte. Diese Gefäßfragmente sind im Zuge der Erdarbeiten, die in diesem Bereich im frühen 7. Jh. stattgefunden haben, gemeinsam mit Keramik des 8.-7. Jhs., aber auch einigen wenigen Fragmenten frühhelladischer Keramik an dieser Stelle in den Boden gelangt: Schilbach 1984, 227 Abb. 2. Im Bereich des Prytaneion im Norden der Altis fanden zuletzt Grabungen von H. Kyrieleis statt. In Schwemmschichten vom Westabhang des Kronoshügels wurde das Stilfragment eines mykenischen Goblets festgestellt. Dazu EDER, Pelopion.

18 HäGg 1968; Renfrew 1985; Kilian 1992; Morgan 1999, 295-304; Pilafidis-Williams 1998, 121-134.

19 Eder 1998 für Argolis, Lakonien, Messenien; Morgan 1999, 365-367; Morgan 2002, 252, 258 für die Korinthia; Papadopoulos Th. 1978-79, 173 zu Achaia. 
ten der mykenischen Welt tiefgreifende politische Veränderungen erfolgten, welche zur Veränderung des Siedlungsmusters in der Peloponnes führten.

\section{Die Landschaft Elis wäHRend deR FRÜHEN Eisenzeit DeS AUSGEHENDEN}

\section{BIS 8. Jhs. v. Chr. (АВB. 1)}

Wenig ist aus den unmittelbar nachmykenischen Epochen des ausgehenden 11. bis 8. Jhs. v. Chr. bekannt, und der traditionelle Begriff der ,Dunklen Jahrhunderte' scheint im Fall von Elis für diese Perioden insofern sinnvoll angewendet, als er den Kenntnisstand der Forschung treffend beschreibt. Die deskriptive Bezeichnung dieser Zeit als ,frühe Eisenzeit" anstelle von ,dunkle Jahrhunderte' setzt sich in der Forschung aber zunehmend durch, und wird auch im vorliegenden Zusammenhang bevorzugt, um den Charakter dieser dynamischen Umbruchszeit nicht unnötig zu vernebeln. Die Zahl der Fundplätze in Elis ist deutlich geringer als für die mykenische Zeit, aber Vorsicht ist angebracht, dies mit einem dramatischen Bevölkerungsrückgang gleichzusetzen. Es ändert sich nämlich der Charakter der Evidenz: Im Laufe der frühen Eisenzeit wurden an anderen Orten neue Begräbnisstätten begründet, die aus einzelnen Gruben- und Steinkistengräbern oder Pithosgräbern bestanden. Diese einfachen Gräber liegen nicht nur an anderen Plätzen als die mykenischen Nekropolen, sondern sind auch bei weitem nicht so auffällig wie die mykenischen Kammergräber. Es ist also damit zu rechnen, daß die schlichten Gräber der frühen Eisenzeit bislang einfach nicht so häufig im Gelände beobachtet wurden wie die z. T. recht imposanten mykenischen Kammergräber.

Auch wenn die Zahl der Fundplätze bis heute gering ist, läßt sich zeigen, daß die Landschaft Elis am Beginn der Früheisenzeit nicht entvölkert und unbewohnt war. Die Funde dieser Epoche stammen in erster Linie aus Gräbern und bestehen zum Großteil aus Keramik, die in der entsprechenden Terminologie als submykenisch, protogeometrisch und geometrisch beschrieben wird. Kennzeichnend für die Situation der Forschung ist, daß es sich primär um verschiedene Streu- und Zufallsfunde aus der Region handelt, die anläßlich von Feldarbeiten und Straßenbaumaßnahmen entdeckt wurden. Nur die systematischen Grabungen aus der Stadt Elis und dem Heiligtum von Olympia, die hier in erster Linie aber der Erforschung der historischen Epochen galten, brachten etwas umfangreichere Fundkomplexe der frühen Eisenzeit zum Vorschein.

Auf dem Gebiet der antiken Stadt Elis (Abb. 2) wurden im Verlauf der griechisch-österreichischen Ausgrabungen zwischen den Jahren 1960 und 1970 insgesamt achtzehn Gräber der Früheisenzeit entdeckt ${ }^{20}$. Die größte Gruppe solcher Gräber wurde bei Ausgrabungen in der Westparodos des hellenistischen Theaters aufgefunden, weitere Anhaltspunkte für die Lage früheisenzeitlicher Friedhöfe bilden einzelne Gräber auf dem Gebiet der späteren Agora und an der Stelle der römischen Ostnekropole im Nordosten des Stadtgebiets von Elis. Es handelt sich durchgehend um Körperbestattungen, zumeist in Einzelgräbern, die aus einfachen, in den Lehm getieften Gruben, in zwei Fällen aber auch aus Steinkisten bestanden. Schmuck und Keramik bildeten den Großteil der bescheidenen Grabbeigaben. Die Wahl einfacher Kisten- und Grubengräber für einzelne Körperbestattungen steht im Kontrast

20 Die Publikation dieser Gräber ist mittlerweile erfolgt: EDER 2001c. 


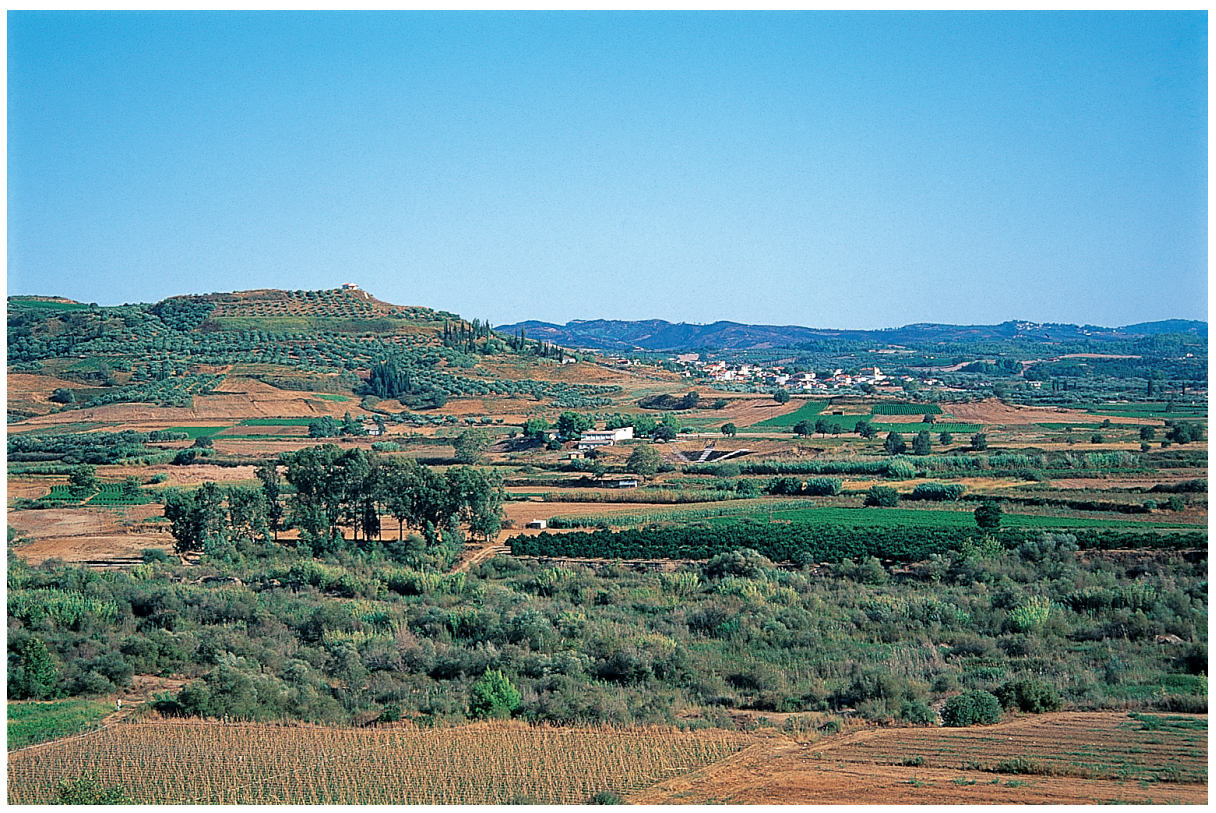

Abb. 2: Stadtgebiet der antiken Stadt Elis von Norden

zur vorherrschenden Grabsitte mykenischer Zeit, mehrfache Beisetzungen in Kammergräbern vorzunehmen ${ }^{21}$.

Anhand des Grabes 1961:7 (Abb. 3) läßt sich der Charakter der Beisetzungen in Elis gut veranschaulichen ${ }^{22}$ : Dieses Grab enthielt ein Skelett in Seitenlage und mit leicht angezogenen Beinen. Eine seitlich gestellte Steinplatte markierte die Grabgrube. Ein Paar Kugelkopfnadeln lag auf den Schultern des Skeletts. Dieser Nadeltyp ist bekannt aus verschiedenen Friedhöfen der Zeit und gehört nach der Typologie von Kilian-Dirlmeier in die submykenische und protogeometrische Zeit ${ }^{23}$. Paarweise getragene Gewandnadeln sind erst in nachmykenischer Zeit regulärer Teil der Gewandtracht geworden und gelten deshalb auch als Hinweis auf einen geänderten Bekleidungsstil in der frühen Eisenzeit. Konservativere Züge zeigt die Keramik aus demselben Grab. Ihre Formen und ihr Dekor stehen in der Töpfertradition der spätmykenischen Keramik der nordwestlichen Peloponnes. Die Bauchhenkelamphore $^{24}$ verrät die Verwandtschaft mit den großen Bauchhenkelamphoren aus Achaia und Elis der Periode SH IIIC, zu deren charakteristischen Ele-

${ }^{21}$ Kisten- und Grubengräber wurden auch in der mykenischen Zeit als Grabstätten verwendet. Dazu zuletzt LewarTowski 2000. In diesem Zusammenhang sind aus der Landschaft Elis z. B. die Grubengräber von Kafkania anzuführen, die der Periode SH IIIC angehören; dazu ParLama 1974, 33-35. Zum Thema Einzel- versus Mehrfachbeisetzungen in Gräbern weist der wichtige Artikel von Dickinson 1983 auf den vielfältigen Befund für mykenische Grabstätten hin, doch mißt er der deutlich merkbaren Veränderung der Grabsitten am Ende der Bronzezeit zu wenig Bedeutung bei. Die Entwicklung von der vorherrschenden Verwendung von Kammergräbern zum überwiegenden Gebrauch von Kisten- und Grubengräbern verlief sicher regional unterschiedlich. Doch die Aufgabe der traditionellen mykenischen Begräbnisstätten in Kammergräbern, die oft über Generationen verwendet worden waren, und die Anlage neuer Friedhöfe sowie die Wahl neuer Grabtypen zu Beginn der Eisenzeit bedarf einer Erklärung, die diesen Diskontinuitäten Rechnung trägt.

${ }^{22} \mathrm{Zu}$ diesem Grab zuletzt EDER 2001c, 19-21.

23 Kilian-Dirlmeier 1984, 69 f. Taf. 7 Nr. 196-197.

24 EDer 2001c, 57-59 Taf. 2c:1; 10a:a.b. 


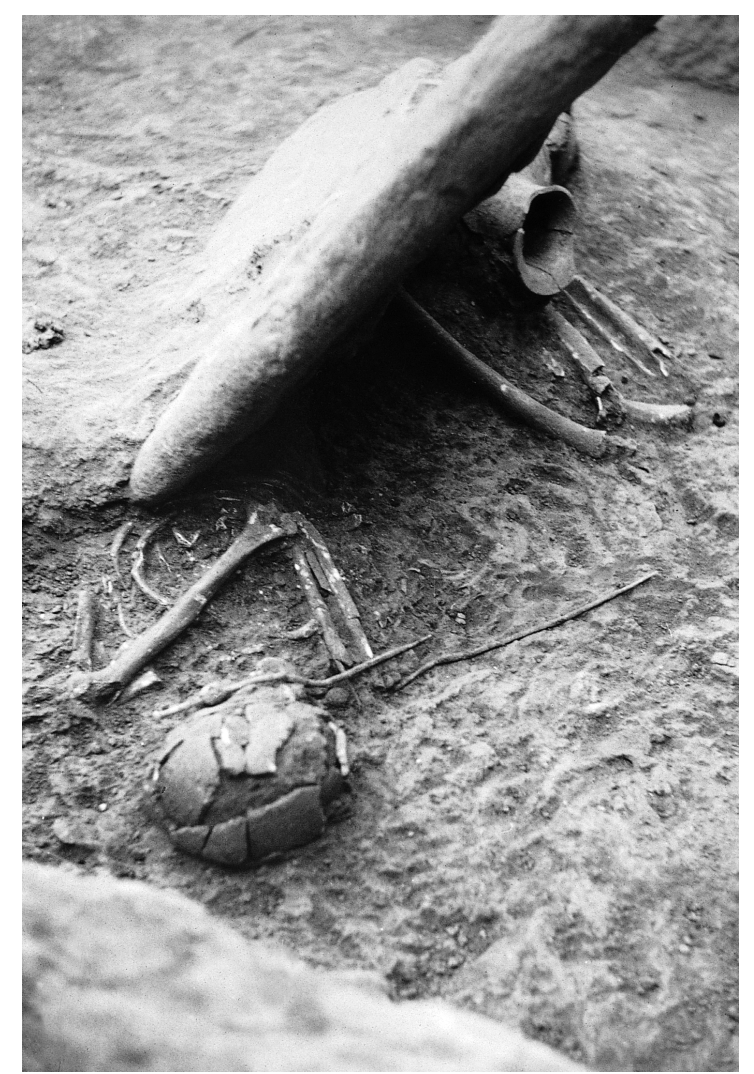

Abb. 3: Stadt Elis: Früheisenzeitliche Beisetzung in Grab 1961:7

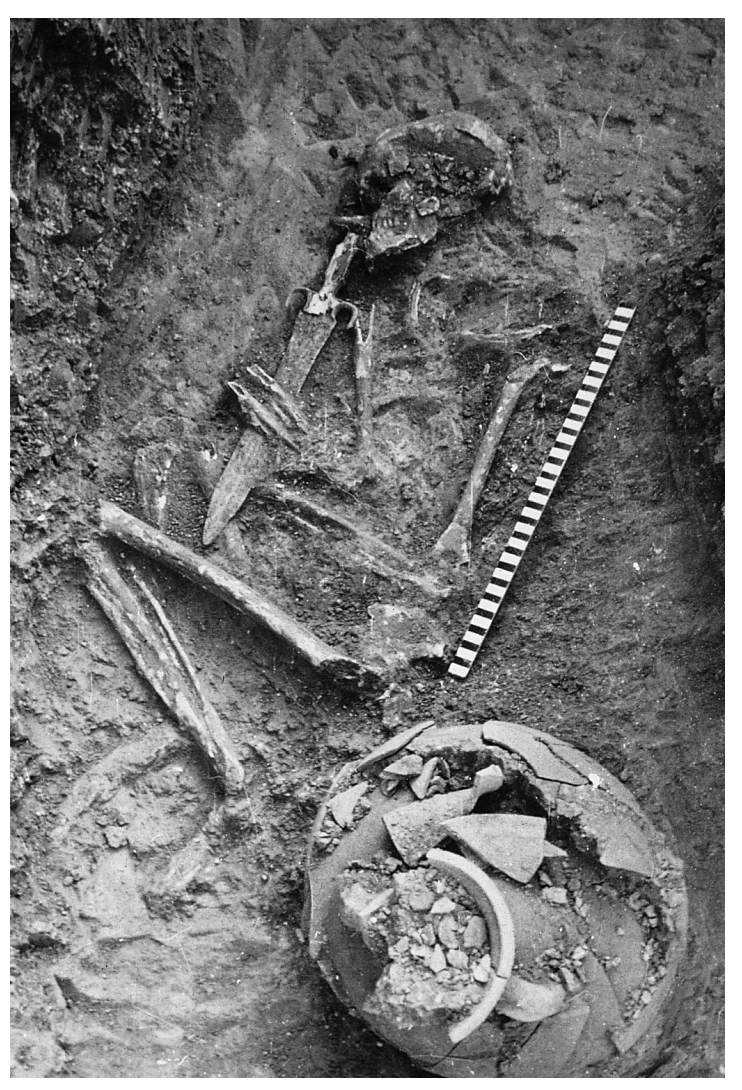

Abb. 4: Stadt Elis: Früheisenzeitliche Beisetzung in Grab 1963:4 
menten beispielsweise die ausgesparte Zone auf der Gefäßschulter mit einer plastischen Warze in der Mitte gehört. Ein jüngeres Vergleichsbeispiel bildet eine protogeometrische Amphore aus Messenien, aus der sog. protogeometrischen Tholos in der Nähe des Palastes von Pylos. Geographisch, stilistisch und auch chronologisch steht die Amphore aus Elis zwischen diesen Vergleichsbeispielen, die darüber hinaus auch die Gemeinsamkeiten in der Keramikproduktion der Westpeloponnes veranschaulichen. Neben dieser Amphore lag eine kleine Lekythos ${ }^{25}$ im selben Grab, die mit einer Dekoration von Dreiecken und Fransen auf der Schulter versehen ist. Die Lekythos löste als Gefäßform in ihrer Funktion als Aufbewahrungsgefäß für Salböle die mykenische Bügelkanne ab. Diese funktionale Verbindung erklärt auch, warum das Muster auf der Lekythos aus Elis eindeutig in der Tradition von Dekorprinzipien steht, wie sie beispielsweise charakteristisch für Bügelkannen der Periode SH IIIC aus der Nachbarlandschaft Achaia sind.

Zwei bronzene Schwerter stammen aus den Gräbern 1963:1 und 1963:4 (Abb. 4) von Elis ${ }^{26}$. In einem Fall handelt es sich um eine Einzelbestattung mit einem sog. Typ G-Schwert (nach der Schwerttypologie von Sandars), im anderen Fall um eine Beisetzung in einem Grab von drei Personen, neben deren Köpfen ein Typ F-Schwert lag. Diese Waffen repräsentieren die traditionellen ägäischen Typen F und Gii der ausgehenden Bronzezeit. Diese Beispiele spätmykenischen Schmiedehandwerks in früheisenzeitlichen Kontexten verdienen angesichts der Tatsache, daß sich bereits gegen Ende der mykenischen Ära der Typus der zentraleuropäischen Naue II-Schwerter in Griechenland durchzusetzen begonnen hatte, besondere Beachtung.

Diese sog. Naue II-Schwerter waren Hieb- und Stichschwerter aus Bronze, die sich im ägäischen Raum seit dem Beginn des 12. Jh. v. Chr. rasch verbreiteten und nach ihrer Umsetzung in Eisen den maßgeblichen Schwerttyp der geometrischen und archaischen Zeit in Griechenland bildeten ${ }^{27}$. Im Vergleich dazu wirken die Schwerter in den Gräbern von Elis sehr altertümlich. Wie eine Reihe von entsprechenden Grabfunden allerdings lehrt, waren die technologisch fortschrittlicheren Naue II-Schwerter in Achaia und in Elis schon in der Periode SH IIIC bekannt ${ }^{28}$. Dies spricht auch dagegen, in den ägäischen Schwertern von Elis ein Indiz für die Rückständigkeit und Provinzialität der Bewohner dieser westpeloponnesischen Landschaft zu erkennen. Die Wahl dieser Waffen als Grabbeigabe könnte hingegen auf den repräsentativen Charakter der Antiquitäten zurückzuführen sein und für den hohen sozialen Status der damit beigesetzten Personen von Elis sprechen. Durch ihren altertümlichen Charakter verbanden sie ihre Träger mit einer mykenischen Vergangenheit. Vor dem fehlenden Hintergrund einer mykenischen Stadtgeschichte konnte es möglicherweise gerade für die Angehörigen der Gruppe, die sich am Beginn des 1. Jts. v. Chr. in Elis als Neusiedler niederließen ${ }^{29}$,

25 EDER 2001c, 68 f. Taf. 2c:2; $12 \mathrm{~b}: a . b$.

26 EDER 2001c, 77-85; EDER 1999.

27 KiLIAN-Dirlmeier 1993, 94-106.

28 Zusammenstellung der Funde bei Eder, Patterns; Papadopoulos Th. 1999; Papadopoulos Th. - Kontorli-Papadopoulou 2001; Papazoglou-Manioudaki 1994. Das erste in der Landschaft Elis gefundene Naue II-Schwert ist ein Zufallsfund aus dem Flußbett des Alpheios: O. VIKaTou, Archaiologikon Deltion 51, 1996, Chron 194 Taf. $62 \varepsilon$.

29 Über die Herkunft der früheisenzeitlichen Siedler in Elis läßt sich aufgrund der Beigaben in den Gräbern keine Aussage treffen; eine Zuwanderung aus der unmittelbaren Umgebung ist ebenso denkbar wie aus den benachbarten Regionen. 
sinnvoll sein, sich mit den Insignien einer vor Ort nicht greifbaren Vergangenheit auszustatten und im Sinn der eigenen Legitimation einzusetzen.

Sog. Kriegergräber, d. h. Beisetzungen mit Waffenbeigaben, gehören zu den elitären Bestattungen der Periode SH IIIC und weisen auf die Kontinuität einer hierarchischen Sozialstruktur über das Ende der mykenischen Paläste um 1200 v. Chr. hinaus. Niederlegung (im Grab) und Zurschaustellung (beim Begräbnis) von Reichtum werden üblicherweise als Ausdruck von Status, Autorität und Macht herrschender Eliten gewertet ${ }^{30}$. Waffen bezeugen in diesem Zusammenhang nicht nur kriegerische Mentalitäten, sondern als Symbole kriegerischer Tüchtigkeit können sie auch zur Legitimation und Entstehung von Status beitragen. Wenn man jedenfalls die zeitgenössische Vasenmalerei als Spiegel der Wertvorstellungen der Periode SH IIIC versteht, liegt die Annahme nahe, daß kriegerische Tüchtigkeit in dieser Zeit nicht nur geschätzt wurde, sondern auch Teil der Identität der herrschenden Elite des 12. Jhs. v. Chr. bildete. Darstellungen von Kriegern im Marsch, auf Streitwagen oder beim Seegefecht gehören nicht umsonst zu den beliebtesten Themen der piktorialen Kunst des 12. Jhs. v. Chr.

Für die Periode, welche auf die letzte Phase der mykenischen Kultur (SH IIIC) folgte, für die Zeit des 11.-10. Jhs. v. Chr., sind derartige Kriegergräber in deutlich geringerer Zahl faßbar. Insofern bilden die beiden Gräber aus Elis mit ihren Schwertbeigaben neben vergleichbaren Bestattungen in Tiryns (Argolis), Elateia (Phokis), Lefkandi (Euböa), Knossos (Kreta) und Zypern wichtige Indizien für die Kontinuität der Beigabensitte. Exotica und Antiquitäten unter den Beigaben könnten hier eine Rolle als Statussymbole gespielt haben, wenn man die ägäischen Schwerter aus Elis und Elateia, den als Typ vereinzelt dastehenden Bronzehelm aus Tiryns oder den babylonischen Goldschmuck der Frauenbestattung neben dem Krieger von Lefkandi bedenkt, selbst wenn unterschiedlich großzügig ausgestattete Begräbnisse unterschiedlich großen Status erkennnen lassen. Überdurchschnittlich reiche Beisetzungen eben wie die im Apsidengebäude von Lefkandi, mit denen das Kriegergrab aus Elis eindeutig nicht konkurrieren kann, bezeugen dann in besonderem Maße durch die Beigabe von Waffen und Pferdegespannen die unmittelbaren Verbindungen, die zwischen Kriegertum und Elitenstatus bestan$\operatorname{den}^{31}$. Diese Situation läßt sich im Übrigen gut mit der Darstellung der herrschenden Eliten in den homerischen Epen und ihres Anspruchs auf militärische Tüchtigkeit vergleichen ${ }^{32}$.

Abgesehen vom Prestigewert der beiden Schwerter aus Elis lassen sich anhand dieser Waffen Verbindungen aufzeigen, welche zwischen verschiedenen Regionen der Peripherie der mykenischen Welt bestanden ${ }^{33}$. Vergleichbare Stücke aus Mittel- und Nordwestgriechenland veranschaulichen nicht nur, daß sich die Stücke aus Elis harmonisch in das Verbreitungsgebiet der Fund G-Schwerter in West- und Mittelgriechenland einfügen, sondern sie illustrieren außerdem die engen Kontakte, die zwischen den Landschaften nördlich und südlich des korinthischen Golfes in spätmykenischer Zeit bestanden

30 Vgl. Kilian-Dirlmeier 1993, 145-148; Deger-Jalkotzy 1999; Voutsaki 1995, 59 f.; VOUTSAKI 1999; WhITLEY 2002.

31 Kilian-Dirlmeier 1998 mit den entsprechenden bibliographischen Verweisen; WhiTLEY 2002; siehe ferner BRÄUning 1995. Lefkandi: PopHAM - CALLIGAS - SACKETT 1993. $\mathrm{Zu}$ den internationalen Beziehungen der mediterranen Eliten des 11.-10. Jhs. siehe CRielaARd 1998; MatthäUs 1998, $140 \mathrm{f}$.

32 VAN WEes 1992, 78-89, 101-108.

33 EDER 1999; EDER 2001c, 77-85. 
haben. So lassen sich aus Ithaka, Elateia in der Phokis und Palioura in Euboia gute Parallelen für das G-Schwert aus Grab 1963:4 anführen. Die Ähnlichkeiten betreffen Griffgestaltung und -dekor, die Form der hörnerartig gebogenen Schwertschultern und den Dekor der Klinge mit schmalen, erhabenen Linien. Diese vier Waffen des Typus G bilden eine in sich homogene Gruppe und sind vielleicht der Produktion einer Werkstätte zuzuordnen, deren Lage in Mittelgriechenland allerdings nur vermutet werden kann. Ähnliches gilt mutatis mutandis auch für das F-Schwert aus Grab 1963:1. Die Verbreitung von Waffen oder wenigstens der Austausch technologischen Wissens muß entlang von Routen, die bis heute das mittelgriechische Festland mit dem Golf von Euboia einerseits und dem korinthischen Golf andererseits verbinden, erfolgt sein.

Analog zu dem Verbreitungsmuster der beiden angeführten Schwerttypen verteilte sich auch der Bernstein, der über Italien aus dem Baltikum importiert wurde, über die Ionischen Inseln im Westen Griechenlands entlang des korinthischen Golfes in den nördlich und südlich angrenzenden Landschaften. Eine kleine Perle gelangte sogar in eines der submykenischen Gräber von Elis (1963:1). Der Meeresarm zwischen Patras und Korinth bildete demnach in der ausgehenden Bronzezeit keine Grenze zwischen der nordwestlichen Peloponnes und dem mittelgriechischen Festland, sondern vielmehr einen der Wege, auf denen abseits der Routen der östlichen Ägäis in den Regionen der sog. Peripherie zwischen Thessalien, den Ionischen Inseln und dem Westen des griechischen Festlands Kommunikation und Austausch von Rohstoffen, Waren und technologischem Know-how erfolgen konnte ${ }^{34}$.

Wichtig ist, daß die früheisenzeitlichen Gräber im Bereich der antiken Stadt Elis die ersten Anzeichen für eine kontinuierliche Besiedlung des Geländes bilden, wo in der Folge die spätere Hauptstadt der Landschaft Elis entstehen sollte ${ }^{35}$. Zwar sind durch Funde früh- und mittelhelladischer Keramik auf der Akropolis und im Bereich des Theaters menschliche Anwesenheit und Besiedlung in Elis während des dritten und frühen 2. Jts. v. Chr. nachgewiesen, doch entscheidend ist, daß mykenische Funde fehlen, die hier eine spätbronzezeitliche Siedlung des 2. Jts. v. Chr. bezeugen könnten. Erst kurz vor Beginn des 1. Jts. v. Chr. zeigen die früheisenzeitlichen Gräber, daß das Gebiet zwischen dem südlichen Peneiosufer und dem Akropolishügel erneut als Siedlungsplatz genutzt wurde.

Die früheisenzeitlichen Gräber bildeten keine geschlossene Nekropole ${ }^{36}$ : Eine Gruppe von vierzehn Gräbern wurde im Westteil des späteren hellenistischen Theaters aufgefunden. Weitere Grabfunde der gleichen Zeitstellung stammen aus dem Nordosten des antiken Stadtgebiets, wo später die römische Nekropole lag. Hier sind bislang zwei Kistengräber und ein Grubengrab dokumentiert. Die Funde aus einem dieser Gräber bestehen z. B. in einer Amphore und einem Askos, der übrigens seine besten Parallelen auch in Funden in den Gebieten jenseits des korinthischen Golfs hat ${ }^{37}$. Diese Funde zeigen, daß die früheisenzeitlichen Gräber nicht auf den Bereich des späteren Theaters beschränkt waren, sondern ein viel größeres Gebiet beanspruchten. Ein

34 EDER, Patterns; zum Bernstein in Elis siehe EDER 2001c, $92 \mathrm{f}$. Zum Bernstein im mykenischen Griechenland: Harding - Hughes-Brock 1974; Harding 1984, 82-87, 305 f.; Hughes-Brock 1993; BouzeK 1993.

35 Dazu vor allem Eder - Mitsopoulos-Leon 1999.

36 Eder - Mitsopoulos-Leon 1999, 6-10; Eder 2001a, 237-239; Eder 2001c, 97-102.

37 EDER 2001c, 35f. zu Grab 1967:25. 
einzeln aufgefundenes Grab innerhalb der Westhalle der Agora ${ }^{38}$ bestätigt den Eindruck, daß innerhalb des Stadtgebiets von Elis mehrere früheisenzeitlichen Gräbergruppen verstreut lagen. Streufunde ohne Kontext wie Nadeln und Gefäße lassen eine ursprünglich weit größere Anzahl von früheisenzeitlichen Gräbern vermuten, als bisher aufgedeckt wurden. Aufgrund der großen Distanzen zwischen den einzelnen Gräbergruppen ist klar, daß die früheisenzeitlichen Gräber keinen geschlossenen Friedhof bildeten, sondern vielmehr zu getrennt angelegten Kleinnekropolen von ein bis zwei Familien gehörten. Entsprechende kleine Siedlungseinheiten, die man sich als weilerartige Kleinsiedlungen vorstellen muß, befanden sich wahrscheinlich in der unmittelbaren Nachbarschaft der Gräber. Diese verstreut liegenden Gruppen von Gräbern bezeichen wahrscheinlich auch den Verlauf eines Weges, der in südwestlich-nordöstlicher Richtung parallel zum Flußtal des Peneios - so wie etwa auch die moderne Straße - verlief ${ }^{39}$.

Abgesehen von den Gräbern auf dem Stadtgebiet der antiken Stadt Elis sind früheisenzeitliche Funde im Peneiostal bislang noch sehr gering. Ein einzelnes Kistengrab mit einem Amphoriskos wurde bei Keramidiá aufgedeckt ${ }^{40}$. Auch wenn es sich um einen bislang allein stehenden Fund handelt, bildet dieses Grab einen wichtigen Hinweis auf die Existenz einer wenigstens kleinen Ansiedlung während der Früheisenzeit. Das Gefäß selbst findet eine gute Parallele in einem Amphoriskos aus Delphi und unterstützt erneut die Annahme von Kontakten zwischen der nordwestlichen Peloponnes und den Gebieten jenseits des korinthischen Golfs während des ausgehenden 11. bis frühen 10. Jhs. v. Chr.

Auch im Alpheiostal ist die früheisenzeitliche Besiedlung bislang nur spärlich bezeugt. Die mykenischen Kammergräber scheinen auch hier - aufgrund der bislang bekannten Funde - nach dem Ende der Periode SH IIIC nicht mehr benützt, sondern als Grabstätten aufgegeben worden zu sein. Die wenigen bisher bekannt gewordenen Fundplätze der Früheisenzeit schließen nicht unmittelbar an die Fundstätten der Spätbronzezeit an.

Bis vor kurzem waren es hauptsächlich die Funde aus den ,Alten Ausgrabungen' in Olympia, die aus dieser Zeit bekannt waren. In dem berühmten Zeusheiligtum (Abb. 5) lag u. a. im Bereich des Pelopion die sog. ,schwarze Schicht', die bereits im 19. Jahrhundert durch die deutschen Ausgrabungen umfassend untersucht wurde. Diese schwarze Schicht erstreckte sich zwischen dem Pelopion und dem Heraion und reichte östlich etwa bis zum Metroon. Adolf Furtwängler beschreibt in seiner grundlegenden Publikation des 4. Bandes des Olympiawerkes diese schwarze Schicht als Ablagerungen fetter Asche mit Knochen, welche Fragmente von Dreifußbeinen, Schmuck und anderen Metallfunden enthielt. Die schwarze Schicht enthielt aber vor allem auch Figurinen aus Bronze und Ton. Aufgrund dieser Evidenz hielt Furtwängler die schwarze Schicht für Überreste von Opfern und Opfergaben, die von einem Altar stammten ${ }^{41}$.

Mehrere Tausend Ton- und Bronzefigurinen, die sowohl Menschen, als auch verschiedene Tiere, vor allem Stiere und Pferde darstellen, stammen aus

38 Eder - Mitsopoulos-Leon 1999, 9 f.; Eder 2001c, 38f. zu Grab 1976:5.

39 Ein ähnliches Siedlungsmuster hat auch A. Papadimitriou im früheisenzeitlichen Tiryns in der Argolis festgestellt: PAPADIMITRIou 1998.

40 EDER 2001a, 239; EDER 2001c, 42.

41 Furtwängler 1890, 2-6, 28 Taf. 10-34; Mallwitz 1988, 81-83 Abb. 6.2 mit einer Karte des Heiligtums, welche die ungefähre Ausdehnung der „schwarzen Schicht“ anzeigt. 


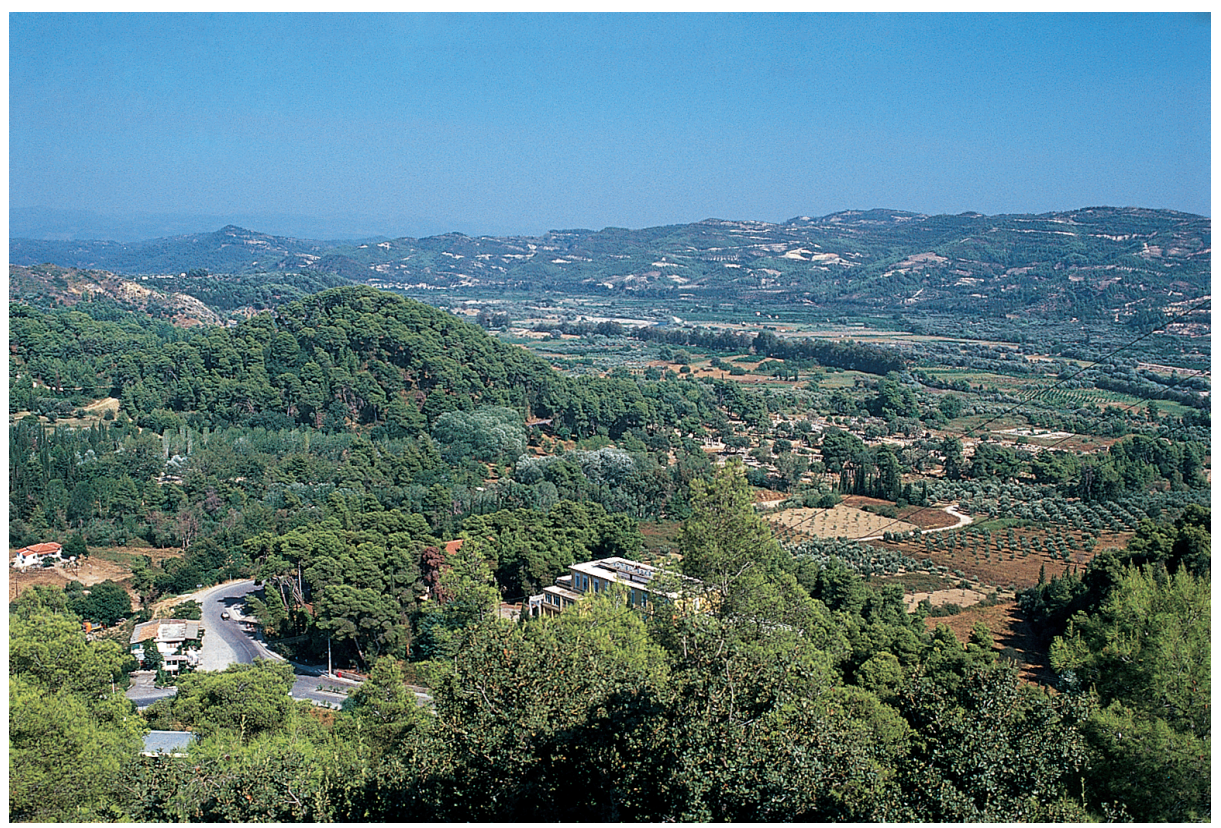

Abb. 5: Olympia und das Alpheiostal

der schwarzen Schicht Olympias. Wolf-Dieter Heilmeyer, der diese Figurinen veröffentlichte, erstellte aufgrund stilistischer Kriterien eine chronologische Abfolge und schlug für die ältesten Figurinen aus Ton ein Datum im späten 10. Jh. v. Chr. vor. Seiner Argumentation zufolge sind die Figurinen aus der schwarzen Schicht alle nachbronzezeitlich und legen eine entsprechende Chronologie für den Kultbeginn im Heiligtum nahe ${ }^{42}$.

Die Frage nach dem Alter des olympischen Heiligtums war vor allem Gegenstand einer kontroversiell geführten Diskussion zwischen Adolf Furtwängler und Wilhelm Dörpfeld. Im Gegensatz zu Furtwängler, der die Anfänge des Kults in Olympia mit der schwarzen Schicht und ihren nachbronzezeitlichen Funden verband, vertrat Dörpfeld die Auffassung von einem bronzezeitlichen Ursprung des Zeuskultes. In seiner Argumentation spielte die Existenz einer prähistorischen Besiedlung innerhalb des späteren Heiligtums eine wichtige Rolle, da er 1908 und 1929 eine Gruppe früh- und mittelbronzezeitlicher Häuser im Norden und Osten des Pelopion aufgedeckt hatte. 1929 grub Dörpfeld auch im Areal des Pelopion und veröffentlichte den Plan eines Tumulus mit Steinkreis im Zentrum des späteren Pelopion. Er war vom hohen Alter des Pelopskultes überzeugt, und er datierte den Tumulus in mykenische Zeit, obwohl er keine einzige mykenische Scherbe zur Unterstützung dieser Chronologie anführen konnte. Er vermutete in diesem Tumulus das Zentrum eines Heroenkultes für Pelops und demzufoge Kultkontinuität bis in historische Zeit. Die unterschiedlichen Positionen von Furtwängler und Dörpfeld bestimmten auch in der Folgezeit die Diskussion über die frühe Ge-

42 HeIlmeYer 1972, 3-6 zum archäologischen Kontext, 10-12, 20, 89f. zur Chronologie der ältesten Figurinen; HeILMEyer 1979, 19-28 und passim zu chronologischen Fragen. Da für die Funde aus der schwarzen Schicht keine stratigraphischen Informationen zu Verfügung stehen, basiert die Datierung der ältesten Figurinen ins 10. Jh. auf der Einordnung der Stücke innerhalb einer stilistischen Entwicklung. Dementsprechend ist der hohe zeitliche Ansatz in der Forschung kritisiert worden: MaLLwiTz 1988,96 . 
schichte des Heiligtums von Olympia. Einerseits war es Alfred Mallwitz, Ausgrabungsleiter in den 1970er Jahren, der den Ausgrabungsbericht Dörpfelds einer kritischen Untersuchung unterzog und sogar die Existenz des Tumulus anzweifelte. Er argumentierte folgerichtig gegen mykenischen Kult im Heiligtum von Olympia. Andererseits war es Hans-Volkmar Herrmann, der auf einige mykenische Scherben hinwies, die innerhalb des Heiligtums gefunden worden waren, und der dementsprechend an der Theorie Dörpfelds vom mykenischen Ursprung des Pelopskults festhielt ${ }^{43}$.

Neue Ausgrabungen waren die einzige Möglichkeit, um die Frage nach dem Beginn und Ursprung des Kults im Heiligtum zu klären. 1986 begannen diese Ausgrabungen unter der Leitung von Helmut Kyrieleis, und konzentrierten sich von 1987 an auf das Gebiet des klassischen Pelopion ${ }^{44}$. Diese Ausgrabungen bestätigten hier die Existenz eines bronzezeitlichen Tumulus, der von einem Steinkreis umgeben war. Dieser Tumulus ist nach den Ergebnissen der neuen Grabungen frühbronzezeitlich (FH II), und es handelte sich um einen einfachen Hügel ohne Gräber, der allerdings ursprünglich mit weiBen Kalksteinplatten bedeckt $\operatorname{war}^{45}$.

Im Zuge der Grabungen wurden auch Teile der schwarzen Schicht aufgedeckt, die zwar von den alten Ausgräbern des 19. Jh. durchsucht und durchhackt, aber nicht an allen Stellen gänzlich abgetragen worden war. Obwohl diese Befunde zum Teil stark gestört waren, konnte festgestellt werden, daß sich die schwarze Schicht aus Asche, Tierknochen, Figurinen, Schmuck und anderen Bronzegegenständen zusammensetzte. Erstmalig wurde Keramik aus dieser Aschenschicht geborgen und dokumentiert. Der archäologische Kontext dieser schwarzen Schicht bestätigt, daß es sich hier um eine Ablagerung von Votiven und Opferresten handelt.

Unter den Steinen der klassischen Pelopionumfriedung waren schmale Stücke der schwarzen Schicht unberührt von den Ausgrabungen des 19. Jhs. in situ stehen geblieben. Die schwarze Schicht begann direkt unterhalb der Steinreihe und saß auf einer sterilen Schicht aus Sand und Lehm. Es ist gewiß, daß es keine stratigraphische Verbindung zum frühbronzezeitlichen Tumulus und jenen frühbronzezeitlichen Gebäuden gibt, die von Dörpfeld ausgegraben worden waren. Die Untersuchung der letzten Reste der schwarzen Schicht ergab außerdem, daß es sich nicht um stratifizierte Schichten, sondern um die angesammelten und vermischten Reste von Votivschutt handelte $^{46}$. Dieser Votivschutt wurde im frühen $7 \mathrm{Jh}$. v. Chr. in einer möglicherweise mehrphasigen Aktion in der Umgebung des Pelopion applaniert, um Geländeunebenheiten auszugleichen.

Da ich die Publikation der Keramik aus diesen Ausgrabungen übernommen habe, kann ich in den vorliegenden Zusammenhang einige Ergebnisse meiner Arbeit einbinden ${ }^{47}$. Charakter und vor allem die chronologische $\mathrm{Zu}$ ordnung der ältesten Keramik aus dem Votivschutt der schwarzen Schicht

43 DöRPFELD 1935, 73-96 zu den prähistorischen Siedlungsresten, 118-124 zum Pelopion, 279-289 gegen die Position von Furtwängler; MaLlwitz 1988, 81-89; HerRmanN 1962; HeRRMANN 1987. Vgl. EDER 2001b, $201 \mathrm{f}$. Zum mykenischen Olympia vgl. auch oben Anm. 14-15.

44 KyRIELEIS 1990, 181, 187 f.; KyRIELEIS 1992. Die abschließende Grabungspublikation wird in der Reihe der Olympischen Forschungen erscheinen.

45 Die früh- und mittelhelladischen Befunde werden von J. Rambach publiziert werden: Vgl. RaMBaCH 2001; RAMBaCH 2002.

46 KYRIELEIS 1990, 187.

47 Eder 2001b; Eder, Pelopion. 
sind für den Charakter und die Chronologie des frühen Kults von Olympia von entscheidender Aussagekraft.

Aufgrund stilistischer Vergleichsbeispiele läßt sich begründen, daß die älteste Keramik aus der schwarzen Schicht in die zweite Hälfte des 11. Jh. zu datieren ist, d. h. in der Fachterminologie in die submykenische bis frühprotogeometrische Periode. Die Keramik aus der schwarzen Schicht insgesamt repräsentiert aber wahrscheinlich den gesamten Zeitraum vom späten 11. Jh. bis zum Anfang des 7. Jhs. v. Chr. Der keramische Befund der schwarzen Schicht enthielt z. B. viele konisch geformte Füße, aber auch Fragmente von Gefäßrändern, gelegentlich mit dem Ansatz von Vertikal- und Horizontalhenkeln. Sie gehören zu verschiedenen Formen von Trinkgefäßen wie Tassen, Bechern mit zwei vertikalen Henkeln oder solchen mit zwei horizontalen Henkeln. Sie sind typisch für die Zeit des 10.-8. Jhs. v. Chr. Diese Art von Trinkgefäßen bildet bei weitem den größten Anteil am Gefäßspektrum. Neben der großen Anzahl von Trinkgefäßen ergänzt eine viel geringere Anzahl von komplementären Gefäßformen das keramische Ensemble. Diese Gefäße bestehen einerseits aus Krateren, Mischgefäßen für Wein, und andererseits aus Kannen, Krügen und Amphoren. Wertet man die Zahlen der entspechenden Keramikfragmente aus, nehmen die offenen Gefäßformen $62 \%$ der Gesamtmenge ein, während $1 \%$ auf geschlossenen Formen und $37 \%$ auf nicht bestimmbare Gefäßtypen entfallen.

Es ist möglich, daß ein Unterschied zwischen diesem Gefäßspektrum und jenem aus einer Siedlung existiert, wo große geschlossene Gefäße üblicherweise etwas zahlreicher sind. Jedenfalls finden sich gute Vergleiche für diese Art der Zusammensetzung des Gefäßspektrums in den Befunden aus den Heiligtümern von Isthmia in der Korinthia und in Kalapodi in Mittelgriechenland ${ }^{48}$. Aus dem keramischen Befund ergibt sich daher eine Vorliebe für Trinkgefäße in Heiligtümern, und diese scheint mir darauf hinzudeuten, daß Trinkgelagen als ritueller aber auch als sozialer Handlung im Rahmen religiöser Feste und Feierlichkeiten eine bedeutende Rolle zukam.

Der Votivschutt aus der schwarzen Schicht bezeugt kultische Aktivitäten, die mit Hilfe der Keramik in die Zeit von der 2. Hälfte des 11. Jhs. bis zum Anfang des 7. Jhs. v. Chr. zu datieren sind. Damit bestätigt sich ungefähr die chronologische Einordnung des Kultbeginns um die Mitte des 11. Jhs. v. Chr., so wie es seinerzeit Heilmeyer aufgrund seiner typologischen Klassifizierung der Terrakottafigurinen postuliert hatte ${ }^{49}$. Dies fügt sich auch gut zu einigen Kleinfunden aus Bronze, wie Fibeln, Nadeln und Ringen, von denen die ältesten in die submykenischen und protogeometrischen Perioden gehören ${ }^{50}$. Es bleibt zu betonen, daß die Votivschicht keine Hinweise auf Kult enthielt, die älter als die Mitte des 11. Jhs. v. Chr. sind. Kultkontinuität seit der Bronzezeit ist in Olympia daher auszuschließen, auch wenn anscheinend bronzezeitliche Traditionen ihren Niederschlag im Kultritual der frühen Eisenzeit gefunden haben, wie im Folgenden zu begründen sein wird.

Im Zuge der neuen Ausgrabungen kamen auch zwei Gefäßschalen von zwei großformatigen Kylikes ans Tageslicht, die stilistisch in die submykeni-

48 Morgan 1999, 152-155 Tabelle I.1, 321-326 zu Isthmia; siehe auch EDER 2001b, 205 Anm. 23 mit dem Hinweis auf die Korrespondenz mit R. Catling, nach dessen Auskunft das früheisenzeitliche Material von Kalapodi ein mit Olympia vergleichbares Gefäßsspektrum aufweist.

49 Siehe oben Anm. 42.

50 Philipp 1981, 23, 34-36, 139-141, 261-263, Taf. 1, 26, 42, 59; danach Herrmann 1987, 435 Abb. 113 d-q. 


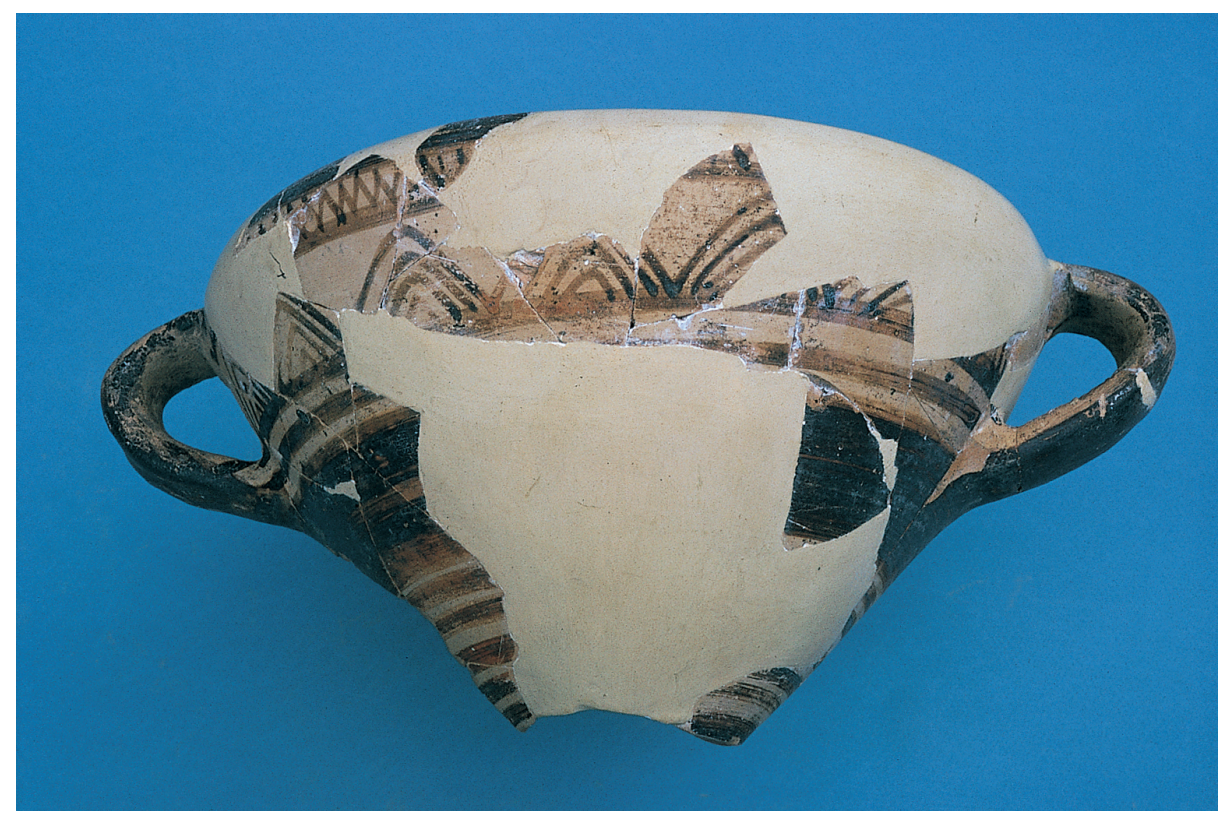

Abb. 6: Olympia: Früheisenzeitliche Kylix aus den Grabungen am Pelopion

sche Zeit gehören (um die Mitte des 11. Jhs.) (Abb. 6). Mit ihrem Durchmesser von etwa 22 bzw. $24 \mathrm{~cm}$ sind sie besonders groß, und daher ist es auch unwahrscheinlich, daß es sich um gewöhnliche Trinkgefäße gehandelt hat. Die Kylix hat eine lange mykenische Tradition in Ritual und Kult. Sie ist eine der typischen mykenischen Vasenformen und war eine der üblichsten und verbreitetsten Trinkgefäßformen mit einem weiten Spektrum an Verwendungsmöglichkeiten. Neben ihrem Gebrauch im profanen Alltag soll in diesem Zusammenhang der Einsatz der Kylix in zeremoniellem und religiösem Kontext betont werden ${ }^{51}$.

Einige wenige Hinweise auf die rituell-zeremonielle Verwendung der Kylix in mykenischer Zeit müssen in diesem Zusammenhang genügen: Im Thronraum des mykenischen Palastes von Pylos in Messenien fanden nach Ausweis der Linear B Tafeln und hunderter Kylikes, die in den unmittelbar anschließenden Lagerräumen gefunden wurden, Bankette statt ${ }^{52}$. Hier schmückte die Illustration einer solchen festlichen Zeremonie passenderweise auch eine Wand: Das bekannte Fresko mit dem auf einem Felsen sitzenden Leierspieler war Teil einer Szene, in der wenigstens zwei Paare von Männern an Tischen sitzen, und ein Rind auf einem Opfertisch liegt ${ }^{53}$. In einen ganz ähnlichen Kontext gehörte das sog. Campstool-Fresko aus dem Palast von Knossos, welches SM III A datiert und als zeremonielle Gelageszene interpretiert wird $^{54}$.

Die Darstellung einer Frau mit Kylix in der Hand auf einem Tonsarg der mykenischen Nekropole von Tanagra in Boiotien verbindet die Kylix mit dem

51 HäGG 1990, 183; GALATY 1999, 28-32.

52 Bankette und Trinkgelage spielten eine bedeutende Rolle in der mykenischen Gesellschaft: WRIGHT 1995; REHAK 1995; HäGG 1996, 607; KILLEN 1994, 71; SÄFLUND 1980; WHITELAW 2001.

53 McCallum 1987, 90 f., 130-132, Taf. X; zusammenfassend Shelmerdine 1999, $20 \mathrm{f}$; SHELMERDINE 2001, $371 \mathrm{f}$. mit Literatur.

54 Mantzourani 1995, 127 Nr. 10, 135. 
Begräbnisritual ${ }^{55}$. Tatsächlich sind die Reste von zerschlagenen Kylikes und anderem Trinkgeschirr in den Zugängen mykenischer Kammer- und Kuppelgräber gefunden worden und legen nahe, daß Trinkrituale Teil der Begräbnisfeierlichkeiten bildeten ${ }^{56}$.

Andere Beispiele bezeugen die Funktion der Kylix im Kult: Die Hand einer unterlebensgroßen Statuette stammt aus dem Heiligtum von Amyklai in Lakonien und mag zur Figur einer Göttin gehört haben. Sie hält eine Kylix und auf ihrem Handrücken windet sich eine Schlange ${ }^{57}$. Außerdem ist auf die Darstellung einer sitzenden Figur auf einer SH IIIC-zeitlichen Amphora aus Tiryns zu verweisen, die in Analogie zu einer religiösen Szene auf einem goldenen Siegelring aus Tiryns gewöhnlich als Gottheit gedeutet wird ${ }^{58}$.

Die Kylix hatte ihren sicheren Platz im Inventar mykenischer Heiligtümer $^{59}$ : Unbemalte Kylikes wurden beispielsweise in Mykene im Kultzentrum und im Lagerraum des Heiligtums des sog. ,Room with the Frescoes“ gefunden. Auch das jüngst publizierte Material des mykenischen Heiligtums der Aphaia auf Aigina veranschaulicht die Bedeutung der Kylix in der mykenischen Kultpraxis. Aufgrund der verfügbaren Evidenz läßt sich behaupten, daß die Kylix innerhalb eines religiös-sakralen Kontextes für den Konsum von Wein während ritueller Feierlichkeiten und für das Ausgießen von Trankopfern verwendet wurde.

SH IIIC Kylikes aus Heiligtumskontexten, etwa in Tiryns und aus dem Tempel von Ag. Irini auf Kea, zeigen, daß diese Tradition den Fall der mykenischen Paläste um 1200 v. Chr. überlebte ${ }^{60}$. Das Gleiche gilt für die Verwendung der Kylix im Begräbnisritual, das auch in Grabkontexten der postpalatialen Periode nachgewiesen ist ${ }^{61}$.

In der mykenischen Peripherie überlebte die Kylix das Ende der mykenischen Zeit. Besonders die Gebiete der westlichen Peloponnes und der Ionischen Inseln haben Beispiele solcher Kylikes produziert. Entsprechende Kylikes kommen aus Ithaka, der Siedlung von Nichoria in Messenien und dem Heiligtum von Amyklai in Lakonien ${ }^{62}$. Bemerkenswert ist vor allem die Serie vollständig erhaltener Kylikes aus dem Heiligtum in der Polishöhle auf Ithaka, unter denen sich große und normalformatige Kylikes protogeometrischer Zeit befinden. Sie sind durch ihre konischen Gefäßschalen und insbesondere durch ihre gerippten Stiele charakterisiert (Abb. 7).

55 Cavanagh - Mee 1995, 49 Abb. 9 Nr. 48; Immerwahr 1995, 115 Abb. 7, 5a nach Praktika 1973, 121 Taf. $10 \beta$.

56 Zu Trinkritualen an mykenischen Gräbern siehe DemaKopoulou 1990, 121 f. Abb. 2223 mit weiteren bibliographischen Angaben.

57 Demakopoulou 1982, 54-56 Taf. 26 Nr. 68.

58 KILIAN 1980, 26-31.

59 Mykene: Moore-Taylour 1999, 32f., 84, Abb. 10-12; Aigina: Pilafidis-Williams 1998, 97, 132f., 186 Liste 2b; Argolis: Kilian 1990, 189 Abb. 4a, 4b, 190, 193; Methana: Konsolaki 2002, 31 f.. Weitere Referenzen bei Albers 1994, 148 mit Anm. 751 Tabelle 10; HäGG 1996, $609 \mathrm{f}$.

60 Tiryns: Kilian 1981, 53, 56; Asine: HägG 1981, 92-93 Abb. 2 P4; Kea: Caskey 1981, 128, 130 Abb. 4, 134.

61 Entgegen der Behauptung von Cavanagh 1998, 111 “...the custom does not preceed the rise of the palaces, and does not seem to have survived their overthrow." Siehe die Beispiele bei EDER 2001b, 207 Anm. 43, die sich auf die westliche Peloponnes beziehen.

62 Gerippte Kylikes in Ithaka: MounTJoy 1999, 475-477 Nr. 20-24; SouyoudzoGLouHaywood 1999, 109-111 Taf. 27-28, 34, 66b mit der älteren Literatur; Amyklai: DeMaKopoulou 1982, 71 f., Taf. 52 Nr. 20-23; Nichoria/Messenien: Coulson 1983, $69 \mathrm{f}$., 82f., 94. Siehe EDER 2001b, 208 mit Anm. 44-51 mit weiteren bibliographischen Verweisen. 


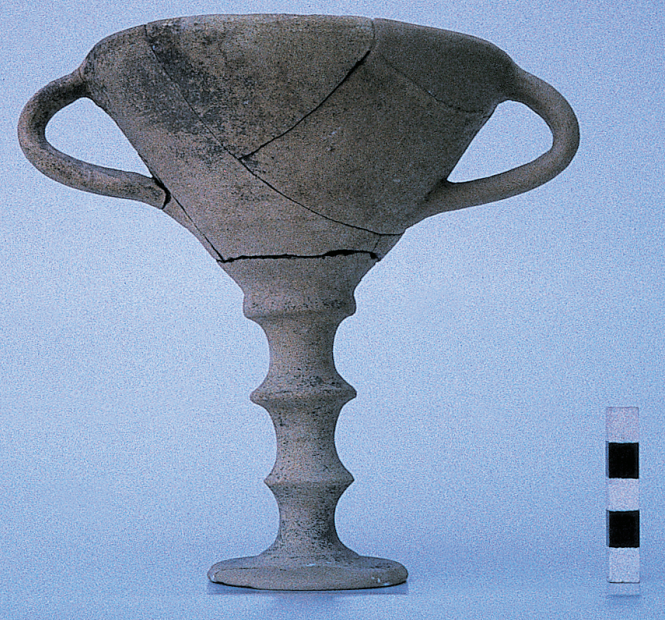

Abb. 7: Ithaka: Früheisenzeitliche Kylix aus der Polishöhle

Eine ganze Reihe solcher gerippter Stiele von Kylikes läßt sich nun, abgesehen von den beiden, bereits erwähnten großformatigen Kylixschalen, auch aus dem Heiligtum von Olympia anführen und als Hinweis auf die Kontinuität der mykenischen Gefäßform im früheisenzeitlichen Kult verstehen ${ }^{63}$. Die Größe einiger dieser Kylixstiele läßt vermuten, daß etliche der früheisenzeitlichen Kylikes aus Olympia eine überdurchschnittliche Größe aufwiesen. Darüber hinaus kennzeichnen stark nach innen gekrümmte Gefäßränder die Kelchschalen der früheisenzeitlichen Kylikes aus Olympia und Ithaka. Insofern waren diese Kylikes ausgesprochen schlecht zum Trinken geeignet, und der nach innen gestülpte Rand verhinderte eher das Ausschwappen der Flüssigkeit. Die tatsächlich oft überdurchnittliche Größe der Gefäße und die zum Trinken eher ungünstige Gestaltung des Gefäßrandes legen daher nahe, daß es sich in diesen Fällen nicht um gewöhnliche Trinkgefäße handelte, sondern daß diese Kylikes in erster Linie zeremoniell-rituell genutzt wurden.

Ein Zusammenhang zwischen den Stücken aus Olympia und Ithaka ist nicht nur aufgrund typologischer Ähnlichkeiten gegeben, sondern auch durch den jeweiligen religiösen Kontext. Aufgrund der Kylikes aus Olympia und jener aus der Polishöhle von Ithaka postuliere ich die Kontinuität der religiösen Symbolik und rituellen Funktion der Kylix von der mykenischen Zeit in die frühe Eisenzeit, selbst wenn der Gebrauch der Kylikes in der frühen Eisenzeit nicht auf ihre religiöse Funktion eingegrenzt werden kann. In diesem Sinne verdankte der frühe Kult in Olympia eine rituelle Tradition der Bronzezeit, obwohl für den Ort keine Kultkontinuität aus der Bronzezeit nachzuweisen ist.

Typologische und stilistische Ähnlichkeiten verbinden die Kylikes mit geripptem Stiel Westgriechenlands mit Kylikes des 11. und 10. Jhs. aus Zypern und weisen auf Beziehungen zur See zwischen diesen Gebieten während der ausgehenden Bronze- und frühen Eisenzeit hin ${ }^{64}$. Sie sind im Rahmen der zy-

${ }^{63}$ EDER 2001b, 206, 208 Taf. 66; EdER, Pelopion.

${ }^{64}$ Siehe EDER, Pelopion; Coulson 1983, 82f. mit Anm. 121-127 zitiert weitere Beispiele. 
prischen Kontakte zu verstehen, die während der ausgehenden Bronze- und frühen Eisenzeit zum westlichen Mittelmeerraum und der Adria bestanden. Kreta und die Ionischen Inseln bildeten innerhalb dieses Netzwerkes verkehrsgeographisch wichtige Kontaktpunkte, ohne daß das gesamte griechische Festland einbezogen gewesen wäre. Diese Beziehungsmuster äußern sich u. a. auch in der Verbreitung von Schwerttypen und Bernsteinperlen und legen nahe, daß die Träger dieser internationalen Beziehungen im Mittelmeerraum die sozialen Eliten des 11.-10. Jhs. waren, die in dieser Zeit auch bemerkenswert ähnliche Statussymbole benutzten ${ }^{65}$. Die gerippten Kylikes aus Olympia lassen vermuten, daß ihre Benutzer zypriotische Gegenstücke kennengelernt oder vermittelt bekommen hatten und werfen ein Licht auf überregionale und mögliche internationale Kontakte tonangebender Besucher des Heiligtums, zu denen die politischen und sozialen Anführer der Region gehört haben müssen. Das Fragment eines zyprischen Dreifußes mit gegossenen Beinen $^{66}$, der aus der Alten Grabung beim Pelopion stammt und in der Forschung traditionell als Erbstück betrachtet worden ist, muß im vorliegenden Kontext vielleicht neu bewertet werden. Die Rolle Ithakas bei der Vermittlung zyprischer Kulturgüter in die Westpeloponnes ist bislang schwer einzuschätzen.

Der zeremoniell-rituelle Kontext für die Verwendung der Kylix gewährleistete wahrscheinlich die Kontinuität der mykenischen Vasenform. Der politische, soziale und wirtschaftliche Hintergrund, welcher die Vorausetzungen für die Bankette und Trinkrituale im Palast von Pylos bildete, veränderte sich nach dem Ende der mykenischen Palastzeit. Doch die Tradition, Kylikes bei Zeremonien und religiösen Handlungen als Trinkgefäße zu verwenden, wurde gerade im Westen Griechenlands beibehalten. Dieser Hinweis auf das Überleben kultischen Brauchtums fügt sich harmonisch zu anderen Elementen religiöser Kontinuität. Einerseits bildeten das Tieropfer und der anschließende Fleischverzehr bereits in mykenischer Zeit einen wichtigen Bestandteil religiöser Feste ${ }^{67}$ und andererseits bestätigen viele griechische Götternamen ${ }^{68}$, die bereits in den bronzezeitlichen Linear B-Texten verzeichnet sind und zu denen auch Zeus gehörte, die konservierende Kraft der Religion. Dies gilt trotz nachweislicher Brüche im archäologischen Befund mykenischer Kultstätten. Religion und Kultausübung sind nicht notwendigerweise an Orte gebunden, sondern in erster Linie an die Menschen, die an Götter glauben und diese verehren.

Einen Hinweis auf die sozialen Rahmenbedingungen, unter denen die Kontinuität religiöser Traditionen erfolgen konnte, bieten Modelle von Streitwagen, -fahrern und Gespannpferden, aber auch Figurinen bewaffneten Krieger unter den Votiven des 8. Jhs. v. Chr. aus Olympia ${ }^{69}$. Der Streitwagen und damit verbunden die Pferdezucht bilden wichtige Elemente an Kontinuität zwischen der frühen Eisenzeit und der mykenischen Vergangenheit Griechenlands. In mykenischer Zeit - folgt man den Linear B-Inventaren von Knossos und Pylos - wurden von den Palästen große Zahlen von Streitwagen hergestellt und verwendet. Das Ende der mykenischen Paläste bedeutete aber nicht

65 Siehe Crielaard 1998; MatthäUs 1998, 140 f.; Eder, Patterns; Eder, Telemachos.

66 Siehe zu diesem Stück zuletzt MatтHÄUs 1985, 309-313 Nr. d Taf. 136, 4.

67 HäGG 1996, 607-611; HäGG 1998; SHELMERDINE 2001, 367-371; WEILHARTNER 2002; Morgan 2002, 254 mit Anm. 20; IsaAKIdou - Halstead - Davis - Stocker 2002; KonSOLAKI $2002,28$.

68 Siehe HäGg 1996, 600 Anm. 9 mit weiterführender Literatur; NeumanN 2002, 44.

69 HeIlmeyer 1972; HeIlmeyer 1979; HeIlmeyer 1982, 68-72; HeIlmeyer 1994. 
das Ende des Streitwagens, sondern aufgrund bildlicher Darstellungen aus dem postpalatialen Griechenland der Periode SH IIIC ist bekannt, daß der leichte zweirädrige Streitwagen, gezogen von zwei Pferden weiterhin ein wichtiges Prestigeobjekt der kriegerischen Führungsschichten des 12. Jhs. v. Chr. bildete ${ }^{70}$. Als nach Jahrhunderten ohne bildliche Darstellungen Streitwagen im 8. Jh. v. Chr. auf attischen Vasenbildern oder in der Gestalt von Bronzefiguren wieder auftauchten, waren die Wagentypen unmittelbar mit den ältesten bronzezeitlichen Vorgängern verwandt ${ }^{71}$.

Produktion und Unterhalt der Streitwagen waren ausgesprochen aufwendig, bedenkt man die komplizierte Herstellung, kontinuierlich notwendigen Reparaturen, angemessene Aufbewahrung, und nicht zuletzt die Aufzucht, Haltung und Ausbildung der dazugehörigen Pferde. Die Kontinuität in Produktion und Verwendung dieses repräsentativen Gefährts spricht dafür, daß sich einige Leute auch nach dem Zusammenbruch der mykenischen Palastherrschaft einen derartigen Luxus leisten konnten, der ihnen eine entsprechenden Status einbrachte. Insofern ist die Existenz sozialer Eliten in der Zeit zwischen 1200 und 700 v. Chr. vorauszusetzen ${ }^{72}$. Figurinen solcher Streitwagen, Streitwagenfahrer und Gespannpferde, aber auch bewaffneter Krieger aus Bronze und Ton aus Olympia bieten nun einen Hinweis darauf, daß, wie C. Morgan postulierte, das Heiligtum wenigstens seit dem 8. Jh. v. Chr. einen Treffpunkt der regionalen Eliten oder Häuptlinge der westgriechischen Landschaften bildete und diesen auch die geeignete Bühne für elitäre Selbstdarstellung bot $^{73}$.

Es ist nicht zu beweisen, allerdings auch nicht auszuschließen, daß zwischen der Weihung von Wagenvotiven und der Abhaltung von Wagenrennen in Olympia ein Zusammenhang bestand, doch ist die Datierung der ältesten olympischen Spiele notorisch schwierig ${ }^{74}$, wenn man das überlieferte Datum von 776 nicht ohne zusätzliche Indizien als historischen Zeitpunkt anerkennen möchte. Wie alt der olympische Wettbewerb der Wagenrennen auch sein $\operatorname{mag}^{75}$, gehören Streitwagen und dazugehörige Pferdegespanne jedenfalls zu den traditionellen Abzeichen einer elitären Weltanschauung, und in diesem Sinne sind die entsprechenden Votivfiguren auch in erster Linie zu verstehen $^{76}$. Es ist daher naheliegend, die im archäologischen Befund zu beobachtende Kontinuität mancher kultureller Phänomene, zu denen auch zeremoniell-religiöse Rituale zu zählen sind, mit den sozialen Eliten der nachpalast-

70 Crouwel 1981, 70-74, 115; GüNTNER 2000, 195-198.

71 Crouwel 1981, 72 f.; Crouwel 1993, 52-65, 106 f.; Crouwel 1995.

72 Kilian-Dirlmeier 1998, 311; WedDe, im Druck. Ich danke M. Wedde, daß er mir das Manuskript seiner Arbeit vor der Drucklegung zur Lektüre überlassen hat.

73 Morgan 1990, 57-105, bes. 90-92; Morgan 1993, 20-24.

74 Zusammenfassend MaLlwitz 1999, 196-199, 220; vgl. auch Sinn 1996, 33-44. Wenn die überlieferte Reihenfolge stimmen sollte, in welcher die einzelnen olympischen Wettbewerbe eingeführt wurden, gab es seit der 25. Olympiade Wagenrennen mit dem Viergespann (680 v. Chr.). Vgl. auch die Anspielung auf die Abhaltung von entsprechenden Wagenrennen „in der göttlichen Elis“ in der Ilias 11, 668-702; dazu CRIELAaRd 1995, 257-259; Kullmann 2002, 101-104.

75 Wagenrennen sind bereits auf mykenischen Vasenbildern bezeugt und mit der kontinuierlichen Existenz des Streitwagens auch für die frühe Eisenzeit vorauszusetzen: Kilian 1980; Crouwel 1981, 142; DeCKer 1987; DeCKer 1992, 129-131. Solche Pferderennen wurden möglicherweise im Rahmen von Leichenspielen abgehalten; sie sind allerdings kein Argument für die Existenz olympischer Wettbewerbe in der Zeit vor dem 8. Jh.

76 Morgan 1990, 90-92. 
zeitlichen Ära zu verbinden, die im Sinne von J. Assmann zu Trägern eines ,kulturellen Gedächtnisses" werden konnten"

Olympia ist somit als bedeutendes früheisenzeitliches Heiligtum der Region zu erkennen, wo einerseits kultische Traditionen Hinweise auf Kontinuitäten aus der Bronzezeit bilden, andererseits die Wahl eines neuen Kultplatzes auf veränderte Bedingungen und neue Siedlungsmuster schließen läßt. Mit Hilfe der Keramik gelingt es wieder, Anhaltspunkte für den Charakter des früheisenzeitlichen Siedlungsmusters in der Region zu gewinnen.

Parallelen für die Keramik aus Olympia stammen vor allem von Fundplätzen der westlichen Peloponnes und den Ionischen Inseln, wie auch die bereits erwähnten Kylikes aus Ithaka illustrieren. Und anhand dieser Parallelen lassen sich die Gefäßformen des insgesamt sehr stark fragmentierten Keramikbestandes auch besser rekonstruieren. Viele Scherben von Bechern aus der schwarzen Schicht haben ihre unmittelbaren Parallelen in der Keramik aus Fundorten in der Umgebung Olympias ${ }^{78}$.

Wie manche dieser Becher einmal ausgesehen haben müssen, veranschaulicht das vollständige Beispiel eines Kantharos, der aus einem Grabkontext des späten 10. Jhs. v. Chr. aus Salmone stammt, einem Ort in etwa $14 \mathrm{~km}$ Entfernung von Olympia ${ }^{79}$. Die entsprechenden Scherben aus Olympia gehören wahrscheinlich zu solchen Bechern mit zwei vertikalen Henkeln und hohem konischen Fuß. Der einfache Dekor besteht aus einem breiten horizontalen Band, das zwischen den Henkeln in der Bauchzone des Gefäßes verläuft und das aus der ansonsten monochromen, meist dunkelbraunen Bemalung ausgespart wurde. Innen verläuft unter dem Rand häufig ein schmaler ausgesparter Streifen. Die Bemalung dieser Becher geht auf Dekortraditionen der spätmykenischen Periode IIIC zurück und zeigt, daß die lokale früheisenzeitliche Keramik in der Tradition der spätmykenischen Keramik steht. Dies betont erneut die Kontinuität eines mykenischen Bevölkerungselements in der Region.

Das vollständige Gefäß aus Salmone veranschaulicht aber auch den lokalen Hintergrund der Keramik aus dem Heiligtum. Gerade anhand der lokal geprägten Keramik läßt sich zeigen, daß das Heiligtum von Olympia in seinen Anfängen ein Heiligtum der umliegenden Region bildete und in den lokal-regionalen Rahmen eingebettet war. Obwohl bislang nur eine geringe Anzahl von Stätten der frühen Eisenzeit in der Umgebung von Olympia bekannt ist, legen einige Gefäße submykenischer bis geometrischer Zeitstellung nahe, daß das Heiligtum nicht in einem Niemandsland existierte ${ }^{80}$.

Aus dem kleinen Ort Gryllos, der südlich von Olympia im Hügelland liegt, stammen zwei Gefäße, ein Becher und ein kleiner Krug. Beide können aufgrund ihres guten Erhaltungszustandes einem Grabinventar zugewiesen werden. Das Trinkgefäß findet wie der Becher aus Salmone ebenfalls in Form und Dekor seine typologische Entsprechung im Material aus Olympia. Gleiches gilt für einen weiteren Becher, der aus einem Grab bei Samikon am südwestlichen Rand des Alpheiostales stammt. Er wurde zusammen mit den Fragmenten von Eisennadeln gefunden und stellt ebenfalls ein Beispiel für die früheisenzeitliche Keramik der Region dar. Einen weiteren Fundplatz mit früheisenzeitlicher Keramik bildet Lasteïka in der Nähe der heutigen Stadt

77 Assmann 1997, 54-59, 70 f. zur Allianz von Herrschaft und Gedächtnis; Assmann 2000, $54 \mathrm{f}$. zur Bedeutung von Festen für das „kulturelle Gedächtnis“.

78 Eder 2001a, 240f.; Eder, Pelopion.

79 Salmone: EDER 2001a, 241; EDER 2001c, 43 f., 73.

80 Eder 2001a; Eder 2001c, 104; Morgan 1999, 380; Morgan 2002, 258. 
Pyrgos. Hier wurde neben einem kleinen Krüglein ein Krug gefunden, dessen handgezogene Halbkreisbögen mit Fransenkranz ein Erbe der spätmykenischen Keramik darstellen. Sowohl Halbkreisbögen als auch die Zickzackleiste am oberen Bildrand finden ihre beste Parallele im Dekor der großformatigen Kylikes von Olympia. Schließlich bezeugen einige Becher und Krüge des 9. und 8. Jhs. v. Chr. aus Trypiti einen weiteren Fundort der frühen Eisenzeit in der Umgebung des Heiligtums von Olympia: Aus gestörten Grabkontexten stammen insgesamt sechs Gefäße, zu denen ein Becher, eine fußlose Tasse, ein Krug und drei Kleeblattkannen gehören. Die einzelnen Gefäße finden auch im Scherbenmaterial von Olympia ihre Entsprechung und tragen zu einem besseren Verständnis des lokalen Keramikspektrums bei ${ }^{81}$.

Diese begrenzte Anzahl von einzelnen Grabfunden bietet Hinweise auf verstreut liegende Siedlungen im Alpheiostal während der Früheisenzeit. Anhand der Keramik aus diesen Gräbern und aufgrund ihrer Ähnlichkeit mit der entsprechenden Keramik aus Olympia läßt sich daher mit Sicherheit behaupten, daß Olympia als Heiligtum der umliegenden Region begann. Insofern ist davon auszugehen, daß die heilige Stätte in ihrer Frühzeit als ein Treffpunkt, Versammlungsplatz und kultischer Mittelpunkt der lokalen Bevölkerung funktionierte, die sich in verschiedenen, verstreuten kleinen Ortschaften innerhalb des Alpheiostales verteilte. Das erste Zentrum des Kults bildete wahrscheinlich der Tumulus aus frühhelladischer Zeit, das spätere Pelopion, dessen Kuppe in der frühen Eisenzeit noch sichtbar gewesen sein dürfte. An diesem Monument der Vergangenheit knüpfte der Kult um die Mitte des 11. Jhs. v. Chr. an.

Den lokalen Charakter des ursprünglichen Heiligtums spiegeln auch die bereits angesprochenen Tausenden von Votivfiguren von Rindern, Pferden und Widdern, die entweder aus Ton oder Bronze gefertigt waren. Die Besucher des Heiligtums und die Stifter der Tierfiguren waren nun wahrscheinlich gerade die wohlhabenden Bewohner der umliegenden fruchtbaren Landschaft, deren Reichtum an Rindern und anderen Herdentieren bereits Homer anschaulich schildert, und der auch den Hintergrund der Sage des Augeias bildet $^{82}$. Der im doppelten Sinne des Wortes sagenhafte Mist, den diese Tiere produzierten, konnte von keinem Geringerem als Herakles selbst beseitigt werden, indem er die Flüsse Alpheios und Peneios durch die Ställe des Augeias leitete.

Aufgrund des vorhandenen Materials ergibt sich für das Ende der Bronzezeit und den Beginn der Eisenzeit ein vorläufiges Bild von den Entwicklungen innerhalb der Landschaft Elis: Nach dem Ende der Periode SH IIIC (ca. $1070 / 50$ v. Chr.) kam es in den lokalen Siedlungsverhältnissen zu einem Umbruch, mit dem eine Veränderung der Begräbnissitten und des Siedlungsmusters einherging. Gegenüber dem Wandel ist andererseits das Fortleben mykenischer Traditionen in der Keramik zu betonen, die Zeugen für die Kontinuität eines mykenischen Elements bilden. Die Ursachen für die wahrzunehmenden Veränderungen sind möglicherweise in einem Umbruch der sozio-politischen Verhältnisse zu suchen und mögen im Zusammenhang mit der überlieferten Siedlungsnahme von Aitolern zu verstehen sein. Jedenfalls bedeutete die Periode am Ende der Bronzezeit und am Anfang der Eisenzeit auch in Elis - wie in den anderen Regionen Griechenlands - ein Zeitalter der Trans-

81 Gryllos, Samikon, Trypiti: EDER 2001a, 241 f.; EDER 2001c, 44 f., 104.

82 Il. 11, 671-681; Od. 4, 635-637; 12, 347. Vgl. Sinn 1996, 14-17; EDER - MitsopoulosLEON 1999, 2 f., $17 \mathrm{f}$. 
formation. In diesen veränderten Bedingungen lagen die Wurzeln für die nachfolgenden Entwicklungen, welche die Geschichte der Landschaft bestimmen sollten. In diese Zeit reichen schließlich die Anfänge einerseits von Elis, der späteren Hauptstadt der Landschaft Elis, und andererseits von Olympia, dem späteren panhellenischen Heiligtum.

Den Abschluß soll ein Ausblick auf die Entwicklung innerhalb der Region bis zum Ende des 8. Jhs. v. Chr. bilden, soweit dies aufgrund der bislang publizierten Funde möglich ist. Während das ausgehende 11. und das 10. Jh. v. Chr. in der Geschichte der Stadt Elis durch die submykenischen und protogeometrischen Gräber und ihre Beigaben gut dokumentiert sind, ist das 9. Jh. v. Chr. in Funden bisher spärlich repräsentiert. Mit Keramik und Kleinfunden, die dem 8. und 7. Jh. v. Chr. zuzurechnen sind, lichten sich die Nebel der sog. Dunklen Jahrhunderte in Elis. Mit diesen Funden läßt sich auch die Brücke zur archaischen Besiedlung der Stadt schlagen und zeigen, daß Elis nicht erst $471 \mathrm{v}$. Chr. - wie die antike Überlieferung lautet - gegründet wurde, sondern schon viel länger, und zwar bereits seit dem ausgehenden 11. Jh. v. Chr. besiedelt war. Zu den Kleinfunden aus Bronze aus Elis zählen die Statuette eines Stiers (Abb. 8) und eines Pferdchens (Abb. 9). Sie gehören ins 9. bis 8. Jh. und haben ihre besten Parallelen in entsprechenden Bronzen aus Olympia. Sie sind der Arbeit einheimischer Bronzehandwerker zuzurechen, die auch im Heiligtum von Olympia nachzuweisen sind. Bereits dem ausgehenden 8. Jh. gehören die Reste eines Kraters und eines Kantharos. Ganz ähnliche Stücke stammen auch aus Achaia und Ithaka, die es erlauben, diese Gefäße in die spätgeometrische Periode (ca. 720-680 v. Chr.) zu datieren ${ }^{83}$.

Ähnliche Kantharoi, die durch feine horizontale Linien in ausgesparten Zonen auf der Lippe und über der Bauchmitte der Gefäße charakterisiert sind, wurden auch in Brunnen in Olympia gefunden. Anhand der Kantharoi läßt sich veranschaulichen, daß im ausgehenden 8 . und frühen 7 . Jh. v. Chr. die Keramik innerhalb der nordwestlichen Peloponnes recht einheitlich geprägt war, und daß entsprechende regionale Kontakte vorausgesetzt werden dürfen ${ }^{84}$.

In diesem Zusammenhang stellt sich als eine Aufgabe für zukünftige Forschungen die Frage nach den Beziehungen zwischen den einzelnen Landstrichen innerhalb der Landschaft Elis und dem Charakter dieser Kontakte zwischen dem 8.-6. Jh. v. Chr. Diese Frage verdient nicht zuletzt vor dem Hintergrund der langedauernden Verbindung von Elis mit Olympia Interesse. Seit wann und in welcher Form standen die Eleier in enger Beziehung zum olympischen Heiligtum? Denn das Datum von 570 v. Chr. gilt zwar traditionell als der Zeitpunkt, als die Eleier die Kontrolle über das Heiligtum gewonnen haben sollen, ist aber nur indirekt aus antiken Quellen erschlossen und alles andere als gesichert ${ }^{85}$.

Damals genoß das Zeusheiligtum von Olympia bereits ein überregionales Ansehen in der griechischen Welt. Weihungen aus lakonischen, argivischen und korinthischen Werkstätten, aber auch aus anderen Regionen Griechenlands wie auch aus Italien im Heiligtum von Olympia legen nahe, daß das Heiligtum seit dem 9. und zunehmend seit dem 8. Jh. v. Chr. einen Besucherkreis von außerhalb der unmittelbaren Landschaftsgrenzen anzog. Einen Teil der

${ }^{83}$ Die Funde wurden erstmals publiziert von EdER - Mitsopoulos-Leon 1999; zur Keramik siehe auch EDER 2001c, 111-113.

84 Siehe auch PaPADOPOUlos J. 2001, 383-407.

85 MölleR 2003. 


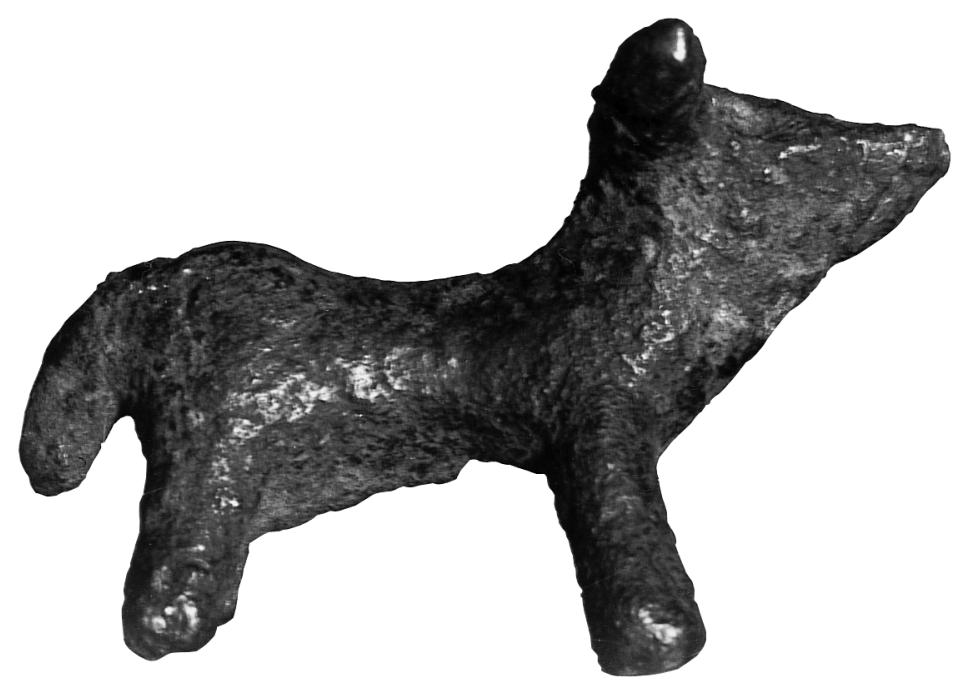

Abb. 8: Elis: Geometrische Stierfigurine aus Bronze (Foto: Archiv ÖAI Athen)

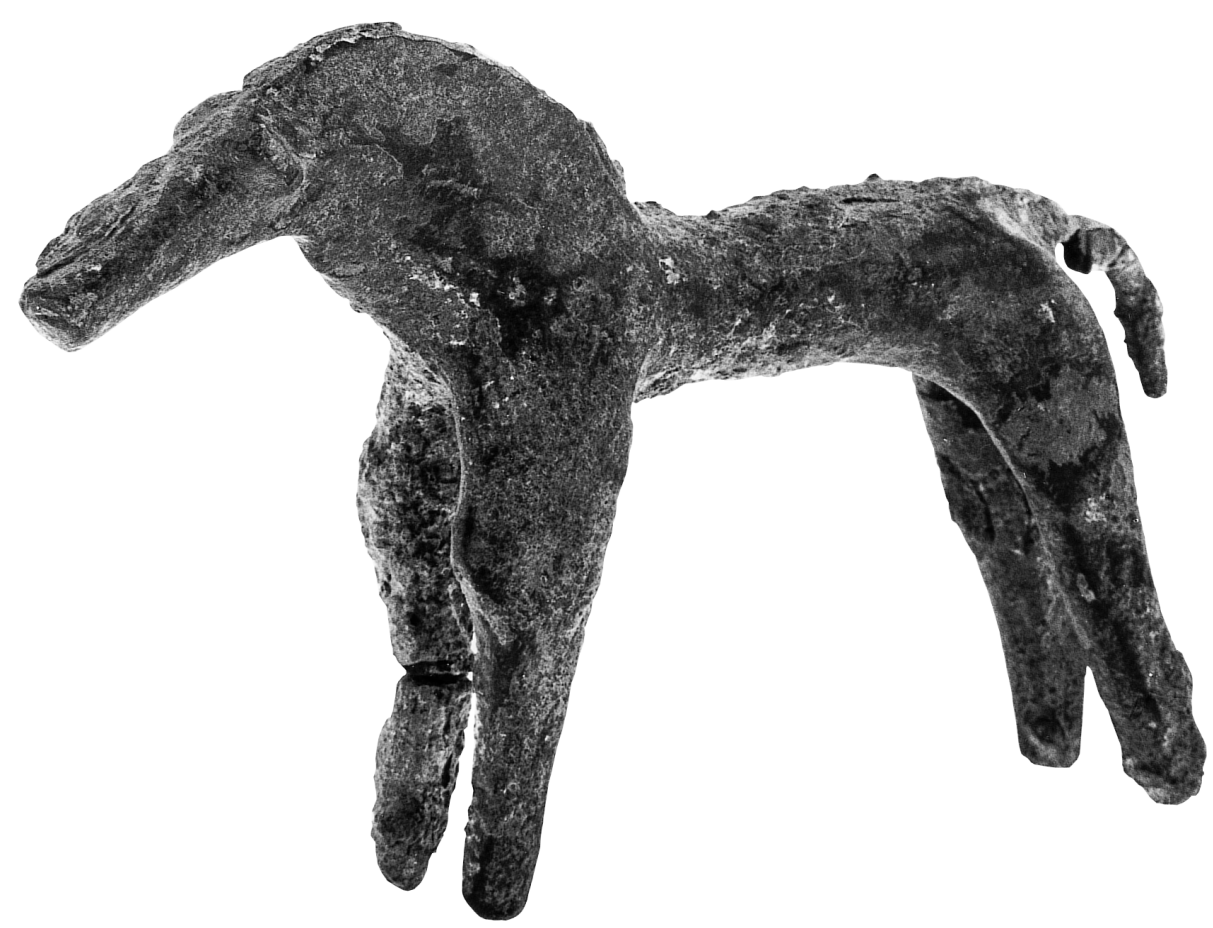

Abb. 9: Elis: Geometrische Pferdefigurine aus Bronze (Foto: Archiv ÖAI Athen)

Attraktion Olympias dürfte neben dem Zeusfest in den dort abgehaltenen Wettkämpfen bestanden haben. Seit dem Ende des 8. Jhs. v. Chr. wurden im Bereich des Stadions in zunehmenden Ausmaß temporäre Brunnen angelegt. A. Mallwitz hat den unmittelbaren Zusammenhang dieser Brunnen mit den olympischen Spielen erkannt und ihre Anlage im späten 8. bis frühen 7. Jhs. v. Chr. in der Nähe des Stadions mit einer steigenden Zahl von Besuchern im Heiligtum im Zuge der Einrichtung der Wettbewerbe und mit entsprechend gesteigertem Wasserbedarf erklärt ${ }^{86}$. Dieser archäologische Befund läßt - an-

86 Mallwitz 1988, 96-100; Mallwitz 1999, 186-199. 
ders als das überlieferten Datum von 776 v. Chr. - vermuten, daß erst seit dem frühen 7. Jh. v. Chr. in Olympia Spiele stattfanden, die einen größeren Besucherkreis anzogen. Erst zu dieser Zeit ergibt dann auch die Anspielung in der Ilias auf die olympischen Spiele einen Sinn. Denn so muß man wohl den Hinweis verstehen, daß Augeias, der Herrscher über Elis, dem Herrscher von Pylos sein zum Wettrennen entsandtes Viergespann raubte ${ }^{87}$.

Auch wenn die Wagenrennen der olympischen Spiele wahrscheinlich nicht vor das 7. Jh. v. Chr. zu datieren sind, so gehen die Ursprünge dieser Disziplin doch in die Bronzezeit zurück. Es waren die Eliten der späten Bronze- und frühen Eisenzeit, die das Fortleben des von zwei Pferden gezogenen Streitwagens gewährleisteten. Das rinder- und pferdereiche Elis bot dazu die optimalen landwirtschaftlichen Voraussetzungen, von denen die Sage von Augeias und seinen Ställen in ihrer besonderen Weise zu erzählen weiß.

\section{BiBLIOGRAPHIE}

ALBERS $1994=$

Assmann $1997=$

Assmann $2000=$

2000.

Aura Jorro - Adrados 1993 = F. Aura Jorro - F. R. Adrados, Diccionario Micénico II, Madrid 1993.

Aura Jorro - Adrados 1999 = F. Aura Jorro - F. R. Adrados, Diccionario Micénico I, Madrid ${ }^{2} 1999$.

Beck - Bouzek 1993 = C. W. Beck - J. Bouzek (Hrsg.), Amber in archaeology. Proceedings of the second international conference on amber in archaeology Liblice, 1990, Prag 1993.

BENNET $1998=$ J. BENNET, The Linear B archives and the kingdom of Nestor, in: J. L. Davis (Hrsg.), Sandy Pylos. An archaeological history from Nestor to Navarino, Austin/Texas 1998, 111-133.

BENNET 1998-99 = J. BENNET, Re-u-ko-to-ro za-we-te. Leuktron as a secondary capital in the Pylos kingdom?, in: J. Bennet - J. Driessen (Hrsg.), ANA-QO-TA. Studies presented to J. T. Killen, Salamanca 19981999 (= Minos 33-34) 11-30.

BENNET $1999=$

BouzeK $1993=$

J. BENNET, The expansion of a Mycenaean palatial center, in: Galaty - Parkinson 1999, 9-18.

J. BouzeK, The shifts of the amber route, in: Beck - Bouzek 1993, 141-146

BRÄUNING $1995=$

A. BRÄUNING, Untersuchungen zur Darstellung und Ausstattung des Kriegers im Grabbrauch Griechenlands zwischen dem 10. Und 8. Jh. v. Chr., Internationale Archäologie, Band 15, Espelkamp 1995.

Caskey $1981=\quad$ M. E. CASKey, Ayia Irini, Kea: the terracotta statues and the cult in the temple, in: Hägg - Marinatos 1981, 127-135.

Cavanagh $1998=$ W. CAvanagh, Innovation, conservatism and variation in Mycenaean funerary ritual, in: K. Branigan (Hrsg.), Cemetery and society in the Aegean Bronze Age, Sheffield 1998, 103-114.

Cavanagh - Mee $1995=$ W. Cavanagh - Ch. Mee, Mourning before and after the Dark Age, in: Morris 1995, 45-61.

87 Siehe oben Anm. 74. 
Cavanagh - Mee 1998 = W. Cavanagh - C. Mee, A private place: death in prehistoric Greece, Studies in Mediterranean Archaeology 125, Göteborg 1998.

CHADwick $1972=\quad$ J. CHADwick, The Mycenaean documents, in: W. A. McDonald G. R. Rapp (Hrsg.), The Minnesota Messenia expedition: reconstructing a Bronze Age regional environment, Minneapolis 1972, 100-116.

Coulson $1983=\quad$ W. D. E. Coulson, The pottery, in: W. A. McDonald - W. D. E. Coulson - J. Rosser (Hrsg.), Excavations at Nichoria in Southwest Greece III: Dark Age and Byzantine occupation, Minneapolis 1983, 61-259.

Coulson - Kyrieleis 1992 = W. D. E. Coulson - H. Kyrieleis (Hrsg.), Proceedings of an international symposium on the Olympic Games, 5-9 September 1988, Athen 1992

Crielaard 1995a = J. P. CrielaArd (Hrsg.), Homeric Questions. Essays in philology, ancient history and archaeology, including the papers of a conference organized by the Netherlands Institute at Athens (15 ${ }^{\text {th }}$ May 1993) Amsterdam 1995.

Crielaard $1995 \mathrm{~b}=\quad$ J. P. Crielaard, Homer, history and archaeology: some remarks on the date of the Homeric world, in: Crielaard 1995a, 201-276.

CrielaARd $1998=\quad$ J. P. CRIElaARD, Surfing on the Mediterranean web: Cypriot long-distance communications during the eleventh and tenth centuries B.C., in: V. Karageorghis - N. Stampolidis (Hrsg.), Proceedings of the international symposium ,Eastern Mediterranean: Cyprus - Dodecanese - Crete $16^{\text {th }}-6^{\text {th }}$ cent. B.C., Rethymnon, 13-16 May, 1997, Athen 1998, 187-206.

Crouwel $1981=\quad$ J. H. Crouwel, Chariots and other means of land transport in Bronze Age Greece, Allard Pierson Series 3, Amsterdam 1981.

Crouwel $1993=$ J. H. Crouwel, Chariots and other wheeled vehicles in Iron Age Greece, Allard Pierson Series 9, Amsterdam 1993.

Crouwel $1995=$ J. H. Crouwel, Chariots in Early Iron Age Greece and in Homer, in: Crielaard 1995a, 309-312.

DECKER $1987=$ W. DeCKer, Die mykenische Herkunft des griechischen Totenagons, in: Th. Eberhard (Hrsg.), Forschungen zur Aegaeischen Vorgeschichte das Ende der mykenischen Welt. Akten des internationalen Kolloquiums 7-8 Juli 1984 in Köln, Köln 1987, 201-230.

Decker $1992=\quad$ W. Decker, Zum Wagenrennen in Olympia - Probleme der Forschung, in: Coulson - Kyrieleis 1992, 129-139.

DeGER-JALKotzy 1999 = S. DegeR-JALKotzy, Military prowess and social status in Mycenaean Greece, in: Laffineur 1999, 121-131.

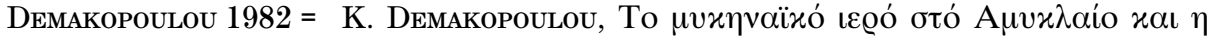

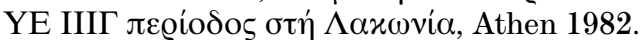

Demakopoulou $1990=$ K. Demakopoulou, Burial ritual in the tholos tomb at Kokla, in: Hägg - Nordquist 1990, 111-123.

Demakopoulou - Crouwel 1998 = K. Demakopoulou - J. H. Crouwel, Some Mycenaean tombs at Palaiokastro, Arcadia, Annual of the British School at Athens 93, 1998, 269-283.

DICKINSON $1977=$ O. T. P. K. Dickinson, The origins of Mycenaean civilisation, Studies in Mediterranean Archaeology 49, Göteborg 1977.

DICKINSON $1983=$ O. T. P. K. Dickinson, Cist graves and chamber tombs, Annual of the British School at Athens 78, 1983, 55-67

EDER $1998=$ B. Eder, Argolis, Lakonien, Messenien vom Ende der mykenischen Palastzeit bis zur Einwanderung der Dorier, Wien 1998.

EDER $1999=$ B. EdER, Late Bronze Age swords from Ancient Elis, in: Laffineur 1999, 443-448.

EDER 2001a $=\quad$ B. EDER, Die Anfänge von Elis und Olympia: Zur Siedlungsgeschichte der Landschaft Elis am Übergang von der Spätbronzezur Früheisenzeit, in: Mitsopoulos-Leon 2001, 233-243.

EDER $2001 b=\quad$ B. EDER, Continuity of Bronze Age cult at Olympia? The evidence from the Late Bronze Age and Early Iron Age pottery, in: 
EDER 2001c $=$

EDER $2003=$

EDER, Patterns $=$

EDER, Pelopion $=$

EDER, Telemachos $=$

GALATY $1999=$

Eder - Mitsopoulos-Leon 1999 = B. Eder - V. Mitsopoulos-Leon, Zur Geschichte der Stadt Elis vor dem Synoikismos von 471 v. Chr.: Die Zeugnisse der geometrischen und archaischen Zeit, Jahreshefte des Österreichischen Archäologischen Instituts 68, 1999, Beibl. 1-40.

A. FurTwäNGLER, Olympia IV: Die Bronzen und übrigen kleineren Funde aus Olympia, Berlin 1890.

M. L. GALATY, Nestor's wine cups: investigating ceramic manufacture and exchange in a Late Bronze Age ,Mycenaean'state, British Archaeological Reports International Series 766, Oxford 1999.

Galaty - Parkinson 1999 = M. L. Galaty - W. A. Parkinson (Hrsg.), Rethinking Mycenaean palaces: new interpretations of an old idea, The Cotsen Institute of Archaeology, monograph 41, Los Angeles 1999.

GÜNTNER $2000=$ W. GüNTNER, Tiryns XII: Figürlich bemalte mykenische Keramik aus Tiryns, Mainz 2000.

HäGG $1968=$

R. HäGG, Mykenische Kultstätten im archäologischen Material, Opuscula Atheniensia 8, 1968, 39-60.

HäGG $1981=\quad$ R. HäGG, The house sanctuary at Asine revisited, in: Hägg - Marinatos $1981,91-94$,

HÄGG $1990=$

HÄGG 1996 = R. HäGG, The role of libations in Mycenaean ceremony and cult, in: Hägg - Nordquist 1990, 177-184.

R. HÄGG, The religion of the Mycenaeans 24 years after the 1967 Mycenological congress in Rome, in: E. de Miro - L. Godart - A. Sacconi (Hrsg.), Atti e memorie del secondo congresso internazionale di micenologia, Roma - Napoli 14-20 ottobre 1991, Rom 1996, 599-612.

HäGG $1998=$ R. HäGG, Ritual in Mycenaean Greece, in: F. Graf (Hrsg.), Ansichten griechischer Rituale, Geburtstagssymposium für Walter Burkert, Castelen bei Basel 15. bis 18. März 1996, Stuttgart 1998, 111-113.

HäGG - Marinatos $1981=$ R. HäGG - N. Marinatos (Hrsg.), Sanctuaries and cults in the Aegean Bronze Age, proceedings of the first international symposium at the Swedish Institute in Athens, 12-13 May 1980, Skrifter utgivna av Svenska Institutet i Athen, $4^{\circ}, 28$, Stockholm 1981.

HÄGG - NoRdqUiST $1990=$ R. HÄGG - G. NoRDQUist (Hrsg.), Celebrations of death and divinity in the Bronze Age Argolid, proceedings of the sixth international symposium at the Swedish Institute in Athens, Skrifter utgivna av Svenska Institutet i Athen, $4^{\circ}$, 40, Stockholm 1990. 
HARDING $1984=$

Harding - Hughes-Brock 1974 = A. Harding - H. Hughes-Brock, Amber in the Mycenaean world, Annual of the British School at Athens 69, 1974, $145-172$.

Heilmeyer $1972=\quad$ W. D. Heilmeyer, Frühe olympische Tonfiguren, Olympische Forschungen 7, Berlin 1972.

HEILMEYER $1979=$ W. D. HeILMEYeR, Frühe olympische Bronzefiguren: Die Tiervotive, Olympische Forschungen 12, Berlin 1979.

HeILMEyer $1982=\quad$ W. D. HeILMEYER, Frühgriechische Kunst: Kunst und Siedlung im geometrischen Griechenland, Berlin 1982.

HeILMeYer $1994=\quad$ W.-D. HeILMeYer, Frühe olympische Bronzefiguren - Die Wagenvotive, in: 9. Olympiabericht, Berlin 1994, 172-208.

HERRMANN $1962=$ H.-V. Herrmann, Zur ältesten Geschichte von Olympia, Athenische Mitteilungen 77, 1962, 3-34.

HERRMANN $1987=$ H.-V. HerRmann, Prähistorisches Olympia, in: H. G. Buchholz (Hrsg.), Ägäische Bronzezeit, Darmstadt 1987, 426-436.

HILLER $1972=$

St. Hiller, Studien zur Geographie des Reiches um Pylos nach den mykenischen und homerischen Texten, Wien 1972.

Hope Simpson $1981=\quad$ R. Hope Simpson, Mycenaean Greece, Park Ridge 1981.

Hughes-Brock 1993 = H. Hughes-Brock, Amber in the Aegean in the Late Bronze Age: some problems and perspectives, in: Beck - Bouzek 1993, 219-229.

Immerwahr $1995=\quad$ S. Immerwahr, Death and the Tanagra larnakes, in: J. B. Carter - S. P. Morris (Hrsg.), The ages of Homer. A tribute to Emily Townsend Vermeule, Austin 1995, 109-121.

ISAAKIDOU - HALSTEAD - DAVIS - StOcKer 2002 = V. IsAakidou - P. HALSTEAD - J. DAvis S. STOCKeR, Burnt animal sacrifice in Late Bronze Age Greece: new evidence from the Mycenaean "Palace of Nestor" at Pylos, Antiquity 76, 2002, 86-92.

KILIAN $1980=\quad$ K. KILIAN, Zur Darstellung eines Wagenrennens in mykenischer Zeit, Athenische Mitteilungen 95, 1980, 21-31.

KILIAN $1981=$

K. Kilian, Zeugnisse mykenischer Kultausübung in Tiryns, in: Hägg - Marinatos 1981, 49-58.

KILIAN $1990=\quad$ K. KILIAN, Patterns in the cult activity in the Mycenaean Argolid: Haghia Triada (Klenies), the Profitis Elias Cave (Haghios Hadrianos) and the Citadel of Tiryns, in: Hägg - Nordquist 1990, 185-197.

Kilian $1992=\quad$ K. Kilian, Mykenische Heiligtümer der Peloponnes, in: H. Froning - T. Hölscher - H. Mielsch (Hrsg.), Kotinos. Festschrift für Erika Simon, Mainz 1992, 10-25.

KILIAN-Dirlmeier 1984 = I. Kilian-Dirlmeier, Nadeln der frühhelladischen bis archaischen Zeit von der Peloponnes, Prähistorische Bronzefunde XIII,8, München 1984.

Kilian-Dirlmeier 1993 = I. Kilian-Dirlmeier, Die Schwerter in Griechenland (außerhalb der Peloponnes), Bulgarien und Albanien, Prähistorische Bronzefunde IV,12, Stuttgart 1993.

KiLIAN-Dirlmeier 1998 = I. Kilian-Dirlmeier, Elitäres Verhalten vom Ende der Bronzezeit bis zum Beginn der Eisenzeit, in: Die Geschichte der hellenischen Sprache und Schrift vom 2. zum 1. Jahrtausend v. Chr.: Bruch oder Kontinuität?, 3. - 6. Oktober 1996 Ohlstadt/Oberbayern, Altenburg 1998, 305-329.

KILLEN $1994=\quad$ J. T. KILLEN, Thebes sealings, Knossos tablets, and Mycenaean state banquets, Bulletin of the Institute of Classical Studies 39, 1994, 67-84.

KonsolaKI $2002=\quad$ E. KonsolaKI, A Mycenaean sanctuary on Methana, in: R. Hägg (Hrsg.), Peloponnesian sanctuaries and cults, proceedings of the ninth international symposium at the Swedish Institute at Athens, 11-13 June 1994, Skrifter utgivna av Svenska Institutet i Athen, $4^{\circ}$, XLVIII (2002) 25-36.

Kullmann $2002=\quad$ W. Kullmann, Homer und das Troia der späten Bronzezeit, in: W. Kullmann, Realität, Imagination und Theorie. Kleine Schriften zu Epos und Tragödie in der Antike, Stuttgart 2002, 97-138. 
KYRIELEIS $1990=$

KYRIELEIS $1992=$

LAFFINEUR $1999=$

LEWARTOWSKI $2000=$

MALLWITZ $1988=$

MALLWITZ $1999=$

MANTZOURANI $1995=$

MATTHÄUs $1985=$

MATTHÄUs $1998=$

McCALlum 1987 =

Mitsopoulos-Leon 2001 = V. Mitsopoulos-Leon (Hrsg.), Forschungen in der Peloponnes. Akten des Symposions anläßlich der Feier „100 Jahre Österreichisches Archäologisches Institut Athen", Athen 5.3.7.3. 1998, Österreichisches Archäologisches Institut, Sonderschriften Band 38, Athen 2001.

MÖLLER $2003=$ A. MöLleR, Elis, Olympia und das Jahr 580 v. Chr.: Zur Frage der Eroberung der Pisatis, in: R. Rollinger - Ch. Ulf (Hrsg.), Griechische Archaik: interne und externe Impulse, Berlin 2003 (im Druck).

Moore - TaYlour 1999 = A. D. Moore - W. D. TaYlour, The temple complex, in: Well Built Mycenae, the Helleno-British excavations within the citadel of Mycenae, 1959-1969, Fasc. 10, Oxford 1999.

MoRGAN $1990=$

MoRGAN $1993=$

Morgan $1999=$

MoRGAN $2002=$

MoRRIS $1995=$

MounTJoY $1999=$

NEUMANN $2002=$

H. Kyrieleis, Neue Ausgrabungen in Olympia, Antike Welt 21.3, 1990, 177-188.

KYRIELEIS, Neue Ausgrabungen in Olympia, in: Coulson R. LafFineur (Hrsg.), Polemos. Le Contexte Guerrier en Égée à nale, Université de Liège 14-17 avril 1998, Aegaeum 19, Liège 1999. naean burial customs, British Archaeological Reports International Series 878, Oxford 2000 .

W. J. Raschke (Hrsg.), The archaeology of the Olympics. The Olympics and other festivals in antiquity, Madison Wisc. 1988,

K. Herrmann (Hrsg.), 11. Bericht über die Ausgrabungen in Olympia: Frühjahr 1977 bis Herbst 1981, Berlin 1999, 186-199. E. Mantzourani, Notes on the depiction of various types of vases nd vessels in Aegean wall-painting, in: Morris 1995, 122-141. zeit, der geometrischen und archaischen Periode auf Cypern, Prähistorische Bronzefunde II, 8, München 1985.

H. МаттнӓUs, Cyprus and Crete in the early first millennium B.C., in: V. Karageorghis - N. Stampolidis (Hrsg.), Proceedings the international symposium ,Eastern Mediterranean: Cy16 May, 1997, Athen 1998, 127-156.

L. McCALlum, Decorative program in the Mycenaean palace of Pylos: the Megaron frescoes, Diss. University of Pennsylvania 1987.

C. Morgan, Athletes and oracles. The transformation of Olympia and Delphi in the eighth century B.C., Cambridge 1990.

C. Morgan, The origins of Pan-Hellenism, in: N. Marinatos - R. Hägg (Hrsg.), Greek sanctuaries: new approaches, London New York 1993, 18-44.

C. Morgan, Isthmia VIII: the Late Bronze Age settlement and Early Iron Age sanctuary, Princeton 1999.

C. Morgan, The origins of the Isthmian festival: points of comparison and contrast, in: H. Kyrieleis (Hrsg.), Olympia 18752000: 125 Jahre Deutsche Ausgrabungen, Internationales Symposion, Berlin, 9.-11. November 2000, Mainz 2002, 251-271. Ch. Morris (Hrsg.), Klados. Essays in honour of J. N. Coldstream, Bulletin of the Institute of Classical Studies Suppl. 63, London 1995.

P. A. MounTJoy, Regional Mycenaean decorated pottery, Rahden/Westf. 1999.

G. NeumanN, Lehnwörter als Indizien für Kulturkontakte: Essay zur Geschichte der frühgriechischen Sprache, in: E. A. Braun-Holzinger - H. Matthäus (Hrsg.), Die nahöstlichen Kul- 


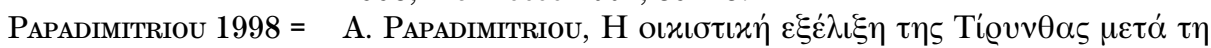

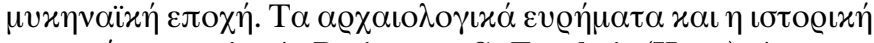

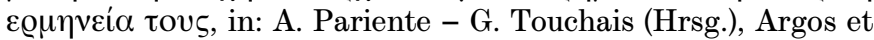
l' Argolide. Topographie et urbanisme, Actes de la Table Ronde internationale, 28. 4.-1. 5. 1990 Athènes - Argos, Recherches Franco-Hélleniques, III, Paris 1998, 117-130.

Papadopoulos J. 2001 = J. K. Papadopoulos, Magna Achaea: Akhaian Late Geometric and Archaic pottery in South Italy and Sicily, Hesperia 70, $2001,373-460$.

Papadopoulos Th. 1978-79 = Th. Papadopoulos, Mycenaean Achaea, Studies in Mediterranean Archaeology 55, Göteborg 1978-79.

Papadopoulos Th. 1999 = Th. Papadopoulos, Warrior-graves in Achaean Mycenaean cemeteries, in: Laffineur 1999, 268-274.

Papadopoulos Th. - Kontorli-Papadopoulou 2001 = Th. J. Papadopoulos - L. KontorLI-PAPAdopoulou, Death, power and troubles in Late Mycenaean Peloponnese. The evidence of warrior-graves, in: P. Fischer (Hrsg.), Contributions to the archaeology and history of the Bronze and Iron Ages in the Eastern Mediterranean. Studies in honour of Paul Åström, Sonderschriften des Österreichischen Archäologischen Instituts, 39, Wien 2001, 127-138.

Papazoglou-Manioudaki 1994 = L. Papazoglou-Manioudaki, A Mycenaean warrior's tomb at Krini near Patras, Annual of the British School at Athens 89, 1994, 171-211.

Parker $1993=\quad$ V. PARKer, Zur Geographie des Reiches von Pylos, Studi micenei ed egeo-anatolici 32, 1993, 41-75.

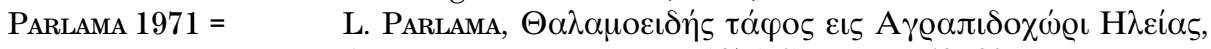
Archaiologike Ephemeris 1971 Chronika, 52-60.

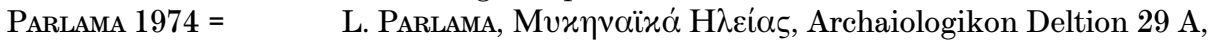
1974, 25-58.

PhILIPP $1981=\quad$ H. PHILIPP, Bronzeschmuck aus Olympia, Olympische Forschungen 13, Berlin 1981.

Pilafidis-Williams 1998 = K. Pilafidis-Williams, The sanctuary of Aphaia on Aigina in the Bronze Age, München 1998.

Popham - Calligas -Sackett 1993 = M. R. Popham - P. G. Calligas - L. H. Sackett, Lefkandi II: The Protogeometric building at Toumba, part 2: the excavation, architecture and finds, Athen 1993.

RAMBACH $2001=\quad$ J. RAMBACH, Bemerkungen zur Zeitstellung der Apsidenhäuser in der Altis von Olympia, in: R. M. Boehmer - J. Maran (Hrsg.), Lux Orientis, Archäologie zwischen Asien und Europa, Festschrift für Harald Hauptmann zum 65. Geburtstag, Rahden/ Westf. 2001, 327-333.

RAMBACH $2002=\quad$ J. RAMBACH, Olympia, 2500 Jahre Vorgeschichte, in: H. Kyrieleis (Hrsg.), Olympia 1875-2000: 125 Jahre Deutsche Ausgrabungen, Internationales Symposion, Berlin, 9.-11. November 2000, Mainz 2002, 177-212.

REHAK $1995=\quad$ P. REHAK, Enthroned figures in Aegean art and the function of the Mycenaean megaron, in: P. Rehak (Hrsg.), The role of the ruler in the Prehistoric Aegean, proceedings of a panel discussion presented at the annual meeting of the Archaeological Institute of America, New Orleans, Louisiana 28 December 1992, Aegaeum 11, Liège - Austin 1995, 95-118.

RENFREW $1985=\quad$ C. RENFREW, The archaeology of cult: the sanctuary at Phylakopi, British School at Athens Suppl. 18, London 1985.

RYSTED $1999=\quad$ E. RYsTED, No words, only pictures: iconography in the transition between the Bronze Age and the Iron Age in Greece, Opuscula Atheniensia 24, 1999, 89-98. 
SÄFLUND $1980=$

SCHILBACH 1984 =

SHELMERDINE $1999=$

SHELMERDINE $2001=$

SINN $1996=$

\section{6.}

Opuscula Atheniensia 13, 1980, 237-246.

J. SсHIввACH, Untersuchungen der Schatzhausterrasse südlich des Schatzhauses der Sikyonier in Olympia, AA 1984, 225-236

C. Shelmerdine, Administration in the Mycenaean palaces: where's the chief?, in: Galaty - Parkinson 1999, 19-24.

C. Shelmerdine, Review of Aegean prehistory VI: the palatial Bronze Age of the Southern and Central Greek mainland, in: T. Cullen (Hrsg.), Aegean prehistory: a review, American Journal of Archaeology Suppl. 1, Boston 2001, 329-381 (= American Journal of Archaeology 101, 1997, 537-585).

U. SInN, Olympia. Kult, Sport und Fest in der Antike, München

Souyoudzoglou-Haywood 1999 = Ch. Souyoudzoglou-Haywood, The Ionian Islands in the Bronze Age and Early Iron Age 3000-800 BC, Liverpool 1999.

VAN WEES 1992 = $\quad$ H. vAN WEES, Status warriors: war, violence and society in Homer and history, Amsterdam 1992.

Ventris - ChadWick 1973 = M. Ventris - J. CHADWICK, Documents in Mycenaean Greek, Cambridge ${ }^{2} 1973$.

VIKATOU 1996-97 =

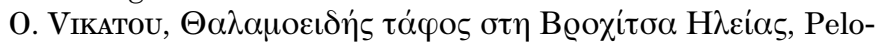
ponnesiaka 22, 1996-1997, 304-313.

VIKATOU $1999 \mathrm{a}=$

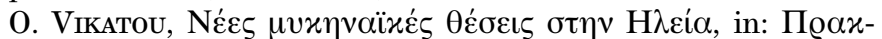

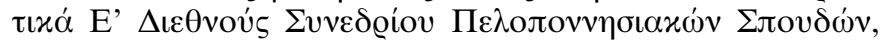

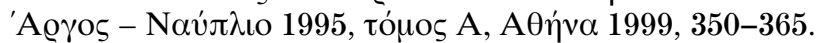

VIKATOU $1999 \mathrm{~b}=$

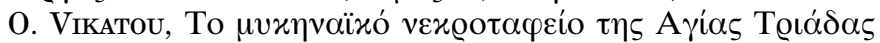

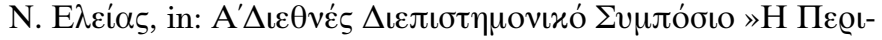

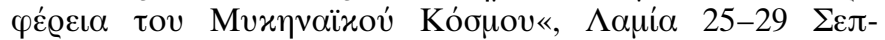

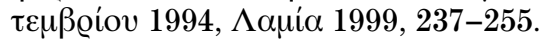

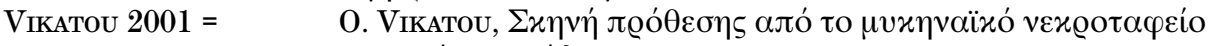

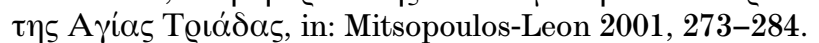

VOUTSAKI $1995=$

S. Voutsaki, Social and political processes in the Mycenaean Argolid: the evidence from mortuary practices, in: R. Laffineur - W.-D. Niemeier (Hrsg.), Politeia. Society and state in the Aegean Bronze Age, proceedings of the $5^{\text {th }}$ International Aegean Conference, University of Heidelberg, Archäologisches Institut 10-13 April 1994, Aegaeum 12, Liège 1995, 55-63.

Voutsaki 1999 = S. Voutsaki, Mortuary display, prestige and identity in the Shaft Grave era, in: Eliten in der Bronzezeit, Ergebnisse zweier Kolloquien in Mainz und Athen, Teil 1, Römisch-germanisches Zentralmuseum, Monographien Band 13,1, Mainz 1999, 103117.

WEDDE, im Druck =

M. WeDDE, Pictorial evidence for partial system survival in the Greek Bronze to Iron Age transition, in: E. Rysted - B. Wells (Hrsg.), Pictorial pursuits. Figurative painting on Mycenaean and Geometric pottery, Symposion at the Swedish Institute at Athens, im Druck.

Weilhartner 2002 = J. Weilhartner, Kultische Festbankette im mykenischen Pylos, in: B. Asamer - P. Höglinger et al. (Hrsg.), Temenos. Festgabe für Florens Felten und Stefan Hiller dargebracht von Schülerinnen und Schülern des Instituts für Klassische Archäologie an der Paris Lodron-Universität Salzburg, Wien 2002, 4552.

WhITELAW $2001=\quad$ T. WHITELAW, Reading between the tablets: assessing Mycenaean palatial involvement in ceramic production and consumption, in: S. Voutsaki - J. Killen (Hrsg.), Economy and politics in the Mycenaean palace states, proceedings of a conference held on 1-3 July 1999 in the Faculty of Classics, Cambridge, Cambridge Philological Society, Suppl. Vol. 27, Cambridge 2001, 51-79.

WHITLEY $2002=\quad$ J. WHITLEY, Objects with attitude: biographical facts and fallacies in the study of Late Bronze Age and Early Iron Age war- 
rior graves, Cambridge Archaeological Journal 12.2, 2002,

WRIGHT $1995=$ 217-232.

J. C. WRIGHT, Empty cups and empty jugs: the social role of wine in Minoan and Mycenaean societies, in: P. McGovern - S. J. Fleming - S. H. Katz (Hrsg), The origins and ancient history of wine, Philadelphia 1995, 287-309.

\section{ABBILDUNGSVERZEICHNIS}

Abb. 1: Karte der Landschaft Elis mit Fundorten des 12. bis 10. Jhs. v. Chr.

Abb. 2: Stadtgebiet der antiken Stadt Elis von Norden

Abb. 3: Stadt Elis: Früheisenzeitliche Beisetzung in Grab 1961:7

Abb. 4: Stadt Elis: Früheisenzeitliche Beisetzung in Grab 1963:4

Abb. 5: Olympia und das Alpheiostal

Abb. 6: Olympia: Früheisenzeitliche Kylix aus den Grabungen am Pelopion

Abb. 7: Ithaka: Früheisenzeitliche Kylix aus der Polishöhle

Abb. 8: Elis: Geometrische Stierfigurine aus Bronze (Foto: Archiv ÖAI Athen)

Abb. 9: Elis: Geometrische Pferdefigurine aus Bronze (Foto: Archiv ÖAI Athen)

Vorgelegt von w. M. SigRID JALKOTZY-DEGER

in der Sitzung am 23. Juni 2003. 
